\title{
Analyse der Lebensqualität von Patienten mit Osteoidosteom vor und nach Behandlung mittels Radiofrequenzablation
}

\author{
Dissertation \\ zur Erlangung des akademischen Grades \\ doctor medicinae (Dr. med.) \\ an der Medizinischen Fakultät \\ der Universität Leipzig
}

eingereicht von: Daniel Seider

geboren am 07.03.1980 in Torgau

angefertigt an der: Klinik und Poliklinik für Diagnostische und Interventionelle Radiologie

Medizinische Fakultät, Universität Leipzig

Betreuer: $\quad$ Prof. em. Dr. med. habil. Thomas Kahn (ehm. Direktor der Klinik und Poliklinik für Diagnostische und Interventionelle Radiologie der Universität Leipzig)

PD Dr. med. habil. Michael Moche (HELIOS Park-Klinikum Leipzig, Leiter der Klinik für Interventionelle Radiologie)

Beschluss über die Verleihung des Doktorgrades vom:

17.12.2019 
„Denke nicht so oft an das, was dir fehlt, sondern an das was du hast."

(Marc Aurel) 


\section{Inhaltsverzeichnis}

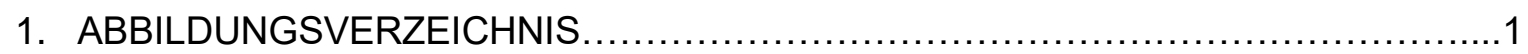

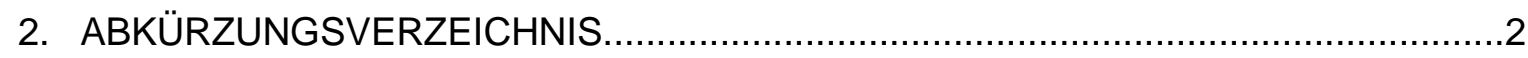

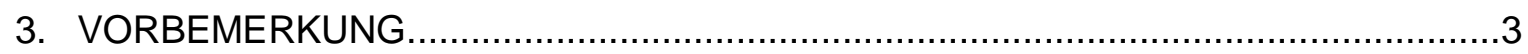

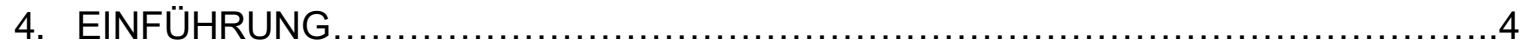

4.1. EPIDEMIOLOGIE UND PATHOPHYSIOLOGIE DES OSTEOIDOSTEOMS.......4

4.1.1. Geschichtlicher Aspekt........................................ 4

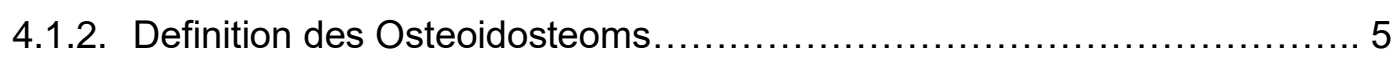

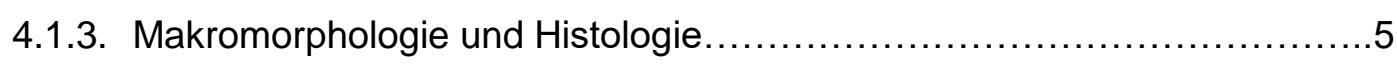

4.1.4. Häufigkeit sowie Alters- und Geschlechtsverteilung .......................

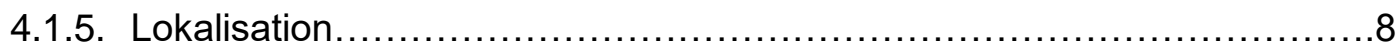

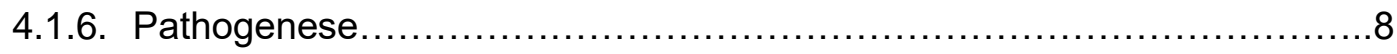

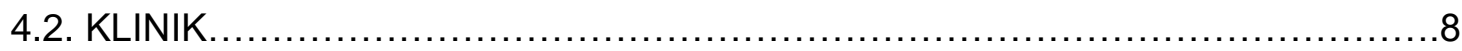

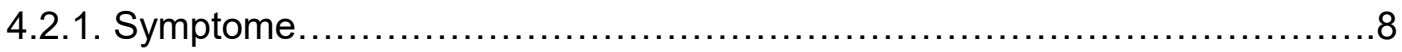

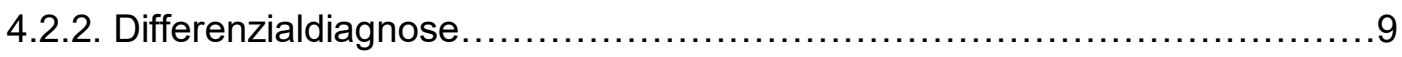

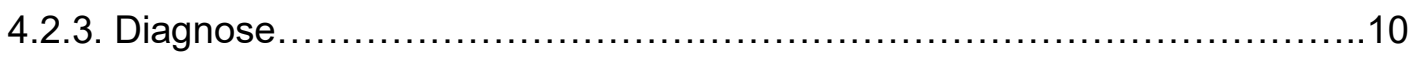

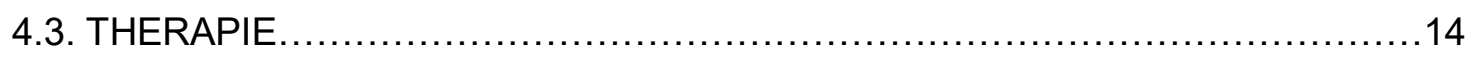

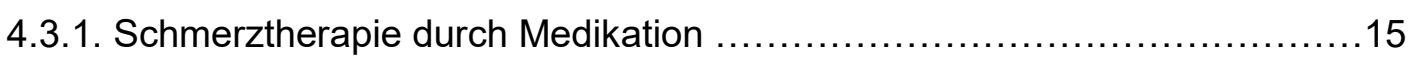

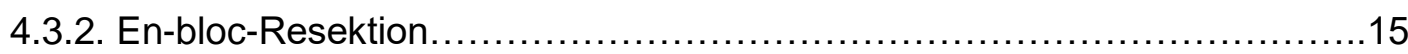

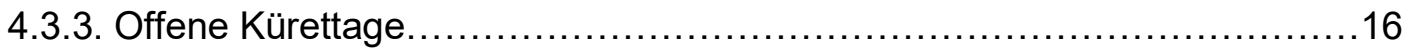

4.3.4. Bildgesteuerte minimal-invasive, perkutane Verfahren.....................16

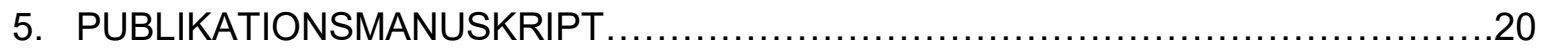

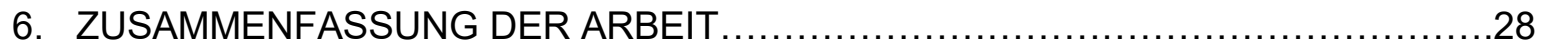

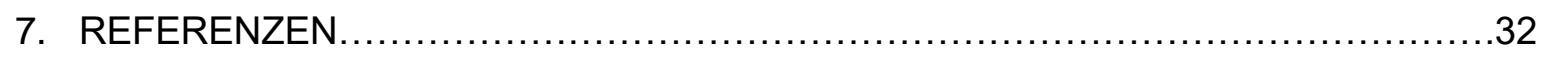

8. ERKLÄRUNG ZUR EIGENSTÄNDIGEN ABFASSUNG DER ARBEIT ................36

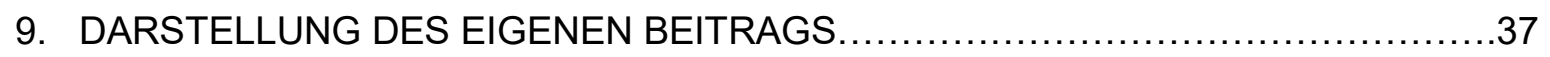

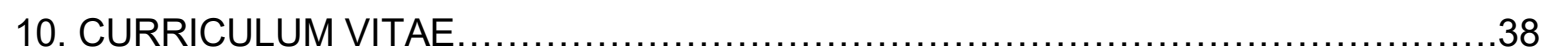

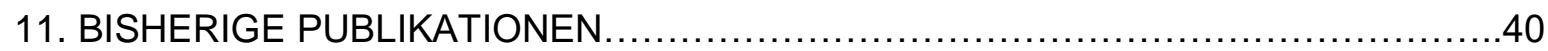

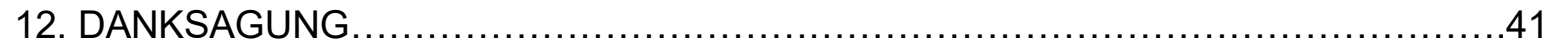


1. Abbildungsverzeichnis

Abb. 1 Histologie des Osteoidosteoms ............................. 6

Abb. 2 Histologie des Osteoblastoms................................ 7

Abb. 3 Osteoidosteom kortikal in der rechten Tibia.

Röntgenaufnahmen

Abb. $4 \mathrm{OO}$ der rechten Tibia, Skelettszintigraphie................... 12

Abb. $5 \mathrm{OO}$ der rechten Tibia, Computertomographie.................. 13

Abb. 6 OO der rechten Tibia, Magnetresonanztomographie......... 14 


\section{Abkürzungsverzeichnis}

$\begin{array}{ll}\text { Abb. } & \text { Abbildung } \\ \text { a.p. } & \text { anterior posterior } \\ \text { ASS } & \text { Acetylsalicylsäure } \\ \text { BSG } & \text { Blutsenkungsgeschwindigkeit } \\ \text { bzW. } & \text { beziehungsweise } \\ \text { CT } & \text { Computertomographie } \\ \text { DD } & \text { Differenzialdiagnose } \\ \text { DM } & \text { Durchmesser } \\ \text { FOV } & \text { field of view } \\ \text { i.v. } & \text { intravenös } \\ \text { MDP } & \text { Methylendiphosphonat } \\ \text { mm } & \text { Millimeter } \\ \text { MRT } & \text { Magnetresonanztomographie } \\ \text { NSAR } & \text { nicht-steroidale Antirheumatika } \\ \text { OO } & \text { Osteoidosteom } \\ \text { OP } & \text { Operation } \\ 99 m \text { Tc } & \text { metastabiles Isotop des Elements Technetium } \\ \text { u.a. } & \text { unter anderem } \\ \text { WHO } & \text { World Health Organization } \\ \text { WK } & \text { Wirbelkörper } \\ \text { z.B. } & \end{array}$




\section{Vorbemerkung}

Es wurde die Form der Publikationspromotion gewählt. Die hierfür erforderliche Veröffentlichung "Analyse der Lebensqualität von Patienten mit Osteoidosteom vor und nach Behandlung mittels Radiofrequenzablation" ist im Februar 2019 als 'Online First'-Artikel im Journal "Der Chirurg“ erschienen und wurde in diese Arbeit eingefügt (siehe 5.).

Die Publikation beinhaltet alle relevanten Daten sowie eine ausführliche Diskussion der Ergebnisse.

\section{Bibliographischer Nachweis der Publikation:}

D. Seider, M. Reinhardt, D. Zajonz, P. Voigt, Th. Kahn, M. Moche

„Analyse der Lebensqualität von Patienten mit Osteoidosteom vor und nach Behandlung mittels Radiofrequenzablation"

Der Chirurg, DOI 10.1007/s00104-019-0818-3

Die Publikation ist als 'Online First'-Artikel verfügbar:

http://link.springer.com/article/10.1007/s00104-019-0818-3 


\section{Einführung}

Die vorgestellte Arbeit untersucht die Lebensqualität von Patienten mit einem Osteoidosteom (OO) vor und nach der Behandlung mittels Radiofrequenzablation (RFA). Des Weiteren werden Aussagen über die Durchführung und Sicherheit dieses Therapieverfahrens gemacht. Folgende Einführung dient der Erläuterung der Epidemiologie und Pathogenese von $\mathrm{OO}$ mit den dazugehörigen klinischen Symptomen. Im zweiten Teil sollen die verschiedenen Therapieverfahren zur Behandlung von $\mathrm{OO}$ vorgestellt werden.

\subsection{Epidemiologie und Pathophysiologie des OO}

\subsubsection{Geschichtlicher Aspekt}

Das Osteoidosteom wurde erstmals 1930 von Bergstrand beschrieben und 1935 von Jaffé als eigene Entität charakterisiert (1). Durch Jaffé erfolgte auch die Identifizierung und Beschreibung z.B. der aneurysmatischen Knochenzyste, des Osteoblastoms, des Riesenzelltumors, des Chondroblastoms, dem nichtossifizierenden Fibrom und der Fibrösen Dysplasie (2). Dadurch wurde eine erste Differenzierung der Knochentumoren geschaffen. So fanden alle Knochengeschwülste eine Zuordnung in Klassifikationssysteme. Dies erfolgte z.B. nach ihrem biologischen Verhalten in primäre maligne, semi-maligne und benigne Tumoren sowie in tumorähnliche Läsionen und Knochenmetastasen. Je nach Histogenese kann die Klassifikation auch in Blutgefäßtumoren, knochenspezifische Tumoren, Knorpeltumoren oder Tumoren des kollagenen Bindegewebes erfolgen (3). In den 1990-iger Jahren übernahm die WHO das heute gängigste Klassifikationsschema und entwickelte es bis zur im Jahre 2013 erschienenen 4. Auflage der Weichteiltumor- und Knochentumorklassifikation weiter (4). Durch detaillierte Definitionen und die Anlage von Tumorregistern gelang es, größere Fallzahlen der eher seltenen Erkrankungen anzulegen und auszuwerten. Jedoch ist die Ätiologie der meisten Knochentumoren sowie die komplexe Kanzerogenese der unterschiedlichen Entitäten weiterhin noch nicht vollständig verstanden $(5,6)$. 


\subsubsection{Definition des OO}

Das OO wurde erstmalig von Henry Lewis Jaffé 1935 als Tumor identifiziert. In den fünf von Jaffé entdeckten Fällen handelte es sich um benigne, osteoblastische Tumoren, die aus Osteoid und atypischem Knochen bestanden und dadurch zur Namensgebung des OOs führten (1).

Die WHO Definition von 2002 in englischer Sprache lautet wie folgt:

"Osteoid osteoma (OO) is defined as a benign bone-forming tumour characterized by small size, limited growth potential and disproportionate pain“ (7).

Diese überwiegend klinisch orientierte Definition spiegelt neben der Benignität die wichtigsten Tumoraspekte wieder, nämlich die geringe Größe und Wachstumstendenz im Verhältnis zur ausgeprägten Schmerzsymptomatik fast aller Patienten. In seltenen Fällen kommt es beim OO zur Selbstheilung mit Sklerosierung des Tumorkerns (Nidus) und Rückbildung der umgebenden Sklerose. Das OO ist in die Kategorie der echten Knochengeschwülste eingereiht und wird als „kleiner Vertreter" des Osteoblastoms behandelt.

\subsubsection{Makromorphologie und Histologie}

Der Nidus ist für das $\mathrm{OO}$ das charakteristische Merkmal. Je nach Ossifikation erscheint dieser weiß-gelblich bis rötlich gefärbt. Histologisch ist der Nidus der eigentliche Tumor. Die Randsklerose stellt dabei nur die Reaktion darauf dar. Der Nidus entspricht mikroskopisch neu gebildetem, ungeordnetem Knochen. Im umgebenden Saum findet man aktive osteoidproduzierende Osteoblasten. Die fehlende Differenzierung verhindert die Bildung lamellären Knochens, wobei die Bälkchenstruktur intakt ist. Der Nidus ist hypervaskularisiert und bildet vasoaktive Substanzen (Prostaglandine) (8). Das Auftreten zahlreicher Nervenfaserendigungen wird für die Schmerzhaftigkeit der Läsion verantwortlich gemacht (9). Die histologische Diagnose gelingt nur, wenn im Untersuchungsmaterial Anteile des Nidus vorhanden sind (Abb.1), denn dieser ist ein differentialdiagnostisches Unterscheidungsmerkmal zum Osteoblastom (Abb. 2), in dem der Nidus fehlt $(8,10)$. Im OO finden sich weder Fettgewebe noch Knorpel, Hämatopoeseinseln, Zell- oder Kernatypien. 


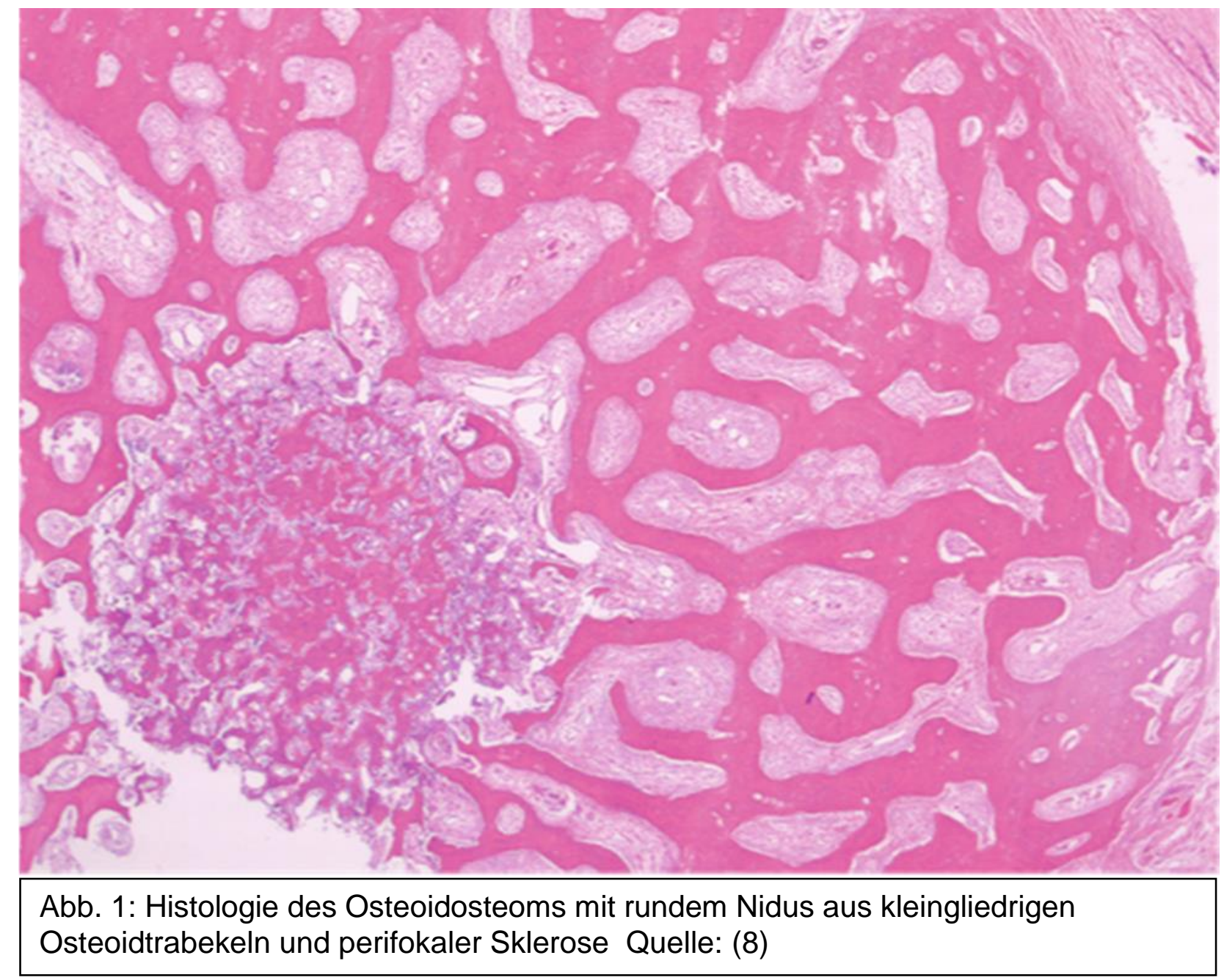




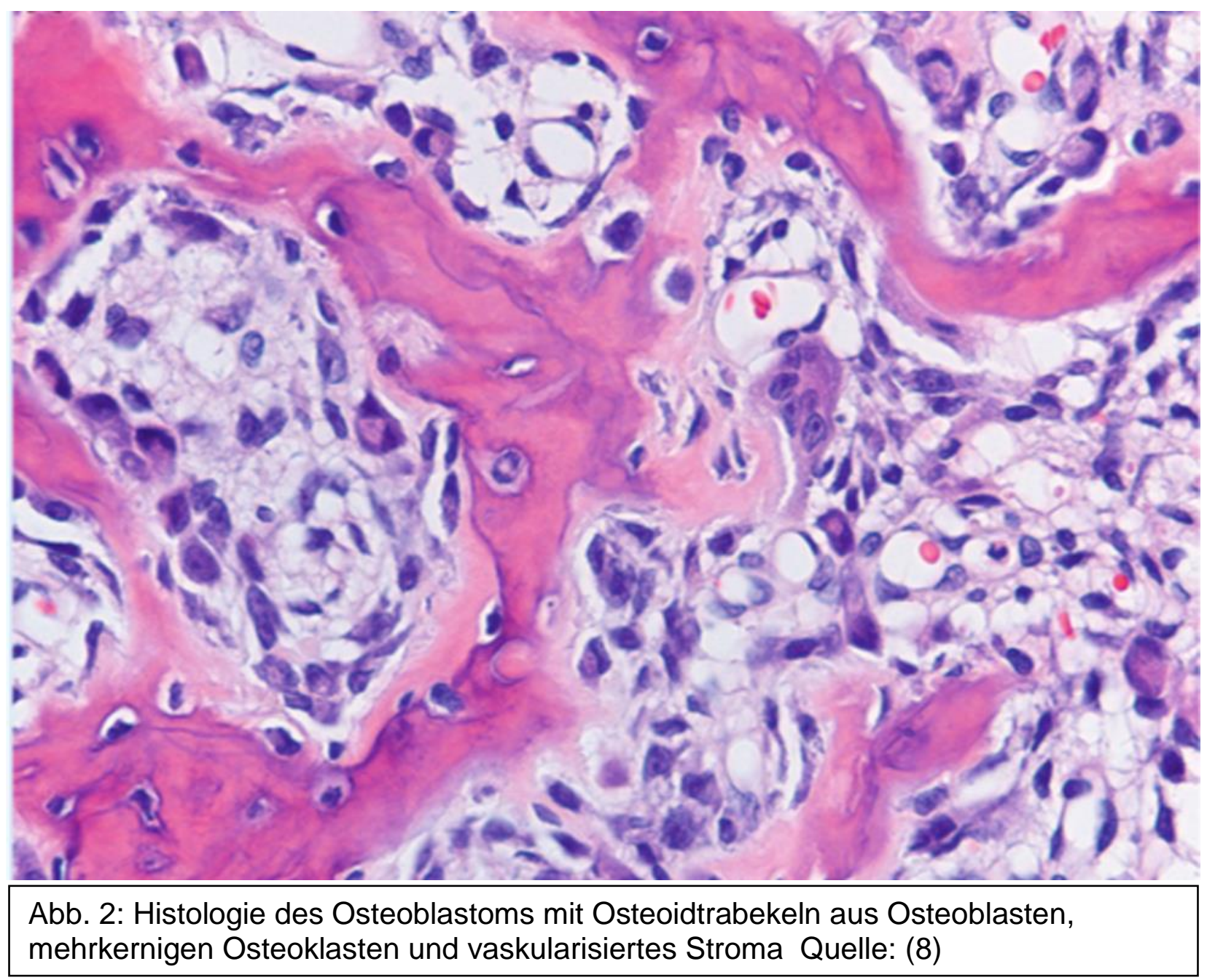

\subsubsection{Häufigkeit sowie Alters- und Geschlechterverteilung}

Das $O O$ zählt mit 3-4\% der primären Knochentumoren und 10-12\% der benignen Knochengeschwülste zu den seltenen Tumoren $(3,5,6,10)$. Die häufige Missdeutung der Tumorsymptomatik führt nicht selten zu einer langen Anamnesedauer mit später Diagnosestellung. Dabei sind durchschnittliche Anamnesedauern von mehr als einem Jahr, in Einzelfällen aber auch deutlich länger, zu verzeichnen (11-13). Männer erkranken 2- bis 4-mal so häufig an einem $\mathrm{OO}$ wie Frauen (14). Das Prädilektionsalter liegt bei etwa $50 \%$ in der 2. Lebensdekade, wobei ca. $90 \%$ der Patienten zwischen 6 und 30 Jahre alt sind. Prinzipiell kann das $\mathrm{OO}$ in jedem Alter vorkommen. Auch bei Erstdiagnose im höheren Lebensalter wird die Entstehung trotzdem im Jugendalter vermutet und dafür ursächlich eine verzögerte Diagnosestellung angenommen (14). 


\subsubsection{Lokalisation}

Grundsätzlich können alle Knochen von einem OO betroffen sein, wobei die Lage im Knochen meist intrakortikal, subkortikal, subchondral oder subperiostal ist. Die langen Röhrenknochen der unteren Extremitäten sind jedoch am häufigsten (ca. 31 $\%$ Femur und $25 \%$ Tibia) betroffen $(5,8)$. Die Verteilung über die Epi-, Meta- und Diaphysenabschnitte ist etwa gleich. Es folgen die Röhrenknochen der oberen Extremitäten und je nach Statistik die Wirbelkörper mit ca. 5-13 \% (5, 14). Bei einer Manifestation an der Wirbelsäule sind meist die hinteren Anteile der WK betroffen (8). Selten ist die Manifestation in flachen Knochen bzw. das Vorkommen von OO mit zwei oder mehreren Nidi sowie das gleichzeitige Auftreten in unterschiedlichen Knochen $(8,15,16)$.

\subsubsection{Pathogenese}

Die Pathogenese ist überwiegend unklar, weshalb hier einige Theorien erwähnt werden sollen. Unstrittig ist die Fehlbildung der Osteogenese im OO, die während des Knochenwachstums auftritt. Für die Theorie, dass es sich womöglich um eine chronische Entzündungsreaktion handelt, spricht das Ansprechen auf NSAR und die Sklerosierung im Knochen (14). Gegen eine infektiöse Entzündung sprechen der fehlende Erregernachweis und das Ausbleiben der typischen Entzündungszeichen, wie Temperaturerhöhung, Leukozytose und beschleunigte BSG (17). Ein anderer Ansatz beschreibt einen posttraumatischen Ursprung, wo die Lokalisation von Trauma und $\mathrm{OO}$ übereinstimmten (18).

\subsection{Klinik}

\subsubsection{Symptome}

Die sehr ausgeprägte, vor allem nächtliche Schmerzhaftigkeit der Tumorregion, welche bei den Patienten zu Ein- bzw. Durchschlafstörungen führt, ist das charakteristischste klinische Symptom des OO. Der Schmerz ist unabhängig von vorausgegangener körperlicher Aktivität und spricht in 25-50 \% der Fälle mit rascher Besserung auf nicht-steroidale Antirheumatika (NSAR, vor allem ASS) an (13, 19). Die Schmerzursache wird unterschiedlich erklärt. Zum einen wird vermutet, dass es durch eine differente Blutfüllung im hypervaskularisierten $\mathrm{OO}$ zu einer 
Druckerhöhung bei zunehmender Durchblutung und damit zur Reizung von Schmerzrezeptoren kommt (4). Des Weiteren führen vom Nidus selbst produzierte Prostaglandine (20) zur Vasodilatation mit anschließender Druckerhöhung und Erregung von Nervenendigungen. Nerveneinsprossungen im Nidus stellen ein besonderes Merkmal des $\mathrm{OO}$ dar. Sie dienen als Unterscheidungsmerkmal zu anderen Tumoren, die bis zur Infiltration des Periosts symptomfrei sind $(9,21)$. Der Schmerz kann an Intensität stark zunehmen. Reflektorisch werden dann Bewegungen vermieden, was zu Muskelatrophie, Mobilitätseinschränkung, Reflexminderung und Fehlhaltung der betroffenen Region führen kann (12, 22). Es gibt aber auch Fälle, bei denen ein Schmerz kaum vorhanden ist oder sogar fehlt. Ist das OO im oder am Gelenk lokalisiert, kann eine Arthritis auftreten. Diese erschwert bzw. verzögert die korrekte Diagnose (23). Ist der Nidus von einem Ödem im Markraum oder Weichteilgewebe umgeben, so kann an tastbaren Skelettabschnitten eine Schwellung wahrgenommen werden. Bei Kindern kann es durch das O० zusätzlich zu Wachstumsstörungen kommen (10).

\subsubsection{Differentialdiagnose}

Als DD zum OO kommt vor allem das Osteoblastom in Betracht. Dieser benigne Tumor, ursprünglich als Riesen-OO bezeichnet, ist dem $\mathrm{OO}$ sehr ähnlich. Histologische Gemeinsamkeit sind aktive Osteoblasten, die Osteoid produzieren und das vaskularisierte Stroma $(10,24)$. Zur richtigen Therapie ist die korrekte Differenzierung unabdingbar. Im Gegensatz zum Osteoblastom wächst das $\bigcirc \bigcirc$ langsam, weniger aggressiv sowie selbstlimitierend und besitzt die deutlichere Schmerzsymptomatik. Das Osteoblastom hat eine höhere Rezidivneigung, wobei sich diese erst nach zwei oder deutlich mehr Jahren manifestiert (3). Des Weiteren treten Rezidive vermehrt in der Wirbelsäule auf. Wichtigstes Differenzierungsmerkmal ist die Tumorgröße. Beim Osteoblastom misst der Nidus in der Regel 1,5 - $2 \mathrm{~cm}$, kann aber auch deutlich größer sein (25).

Röntgenologisch kommt differentialdiagnostisch zum $\mathrm{OO}$ noch eine entzündliche Erkrankung (z.B. Brodie-Abszess), die Stressfraktur oder das Enostom in Frage.

Der Brodie-Abszess kann im Röntgen, CT und MRT dem OO sehr ähnlich sein. Abszesskalzifikationen können dabei mit Verkalkungen im Nidus verwechselt werden. Der Nachweis eines Fistelganges kann zur Differenzierung der Entitäten beitragen. In der Knochenszintigraphie kommt es in der Abszesshöhle zur 
verminderten Radionuklid-Anreicherung, im Gegensatz zum OO mit Double-DensitySign. Im Biopsat ist im Brodie-Abszess häufig das Bakterium Staphylococcus aureus nachweisbar.

Bei der Stressfraktur findet sich röntgenologisch eher eine Aufhellungslinie, die senkrecht oder spitzwinklig zur Kortikalis verläuft (26).

Beim verkalkten Nidus muss als DD ein Enostom (Kompaktainsel) berücksichtigt werden. Das Enostom zeigt im spongiösen Knochen radiäre Streifen, welche mit den Trabekeln des regulären Knochens verschmelzen. Das Knochenszintigramm weist in $90 \%$ keine vermehrte Speicherung auf (26).

\subsubsection{Diagnose}

Das OO fällt in erster Linie durch den starken Nachtschmerz auf, welcher den Hinweis auf ein OO liefern kann. Der Schmerz spricht sehr gut auf die Gabe von ASS oder anderen NSAR an und lässt dann rasch nach. Die Schmerzsymptomatik kann bereits zu einem sehr frühen Zeitpunkt auftreten, zu dem sich der Tumor aufgrund seiner geringen Größe der radiologischen Detektion noch entzieht.

Bildgebende Verfahren zur Diagnose des OO:

\section{Röntgen:}

Die Verdachtsdiagnose kann durch Röntgenaufnahmen in 2 Ebenen gesichert werden (Abb. 3). Charakteristisch ist eine scharf begrenzte, rundlich-ovale Aufhellung (Osteolyse), welche den Nidus darstellt. Eine winzige zentrale Verschattung repräsentiert den Sklerosekern. Eine weitere Transparenzminderung ist um den Nidus lokalisiert und repräsentiert den durch Knochenneubildung hervorgerufenen sklerotischen Randsaum. Aufgrund von Überlagerungen mit der Sklerosezone bzw. anderen knöchernen Strukturen kann es vorkommen, dass die Darstellung des Nidus nicht gelingt (27-29) oder das OO im Röntgenbild vergrößert erscheint (14). 


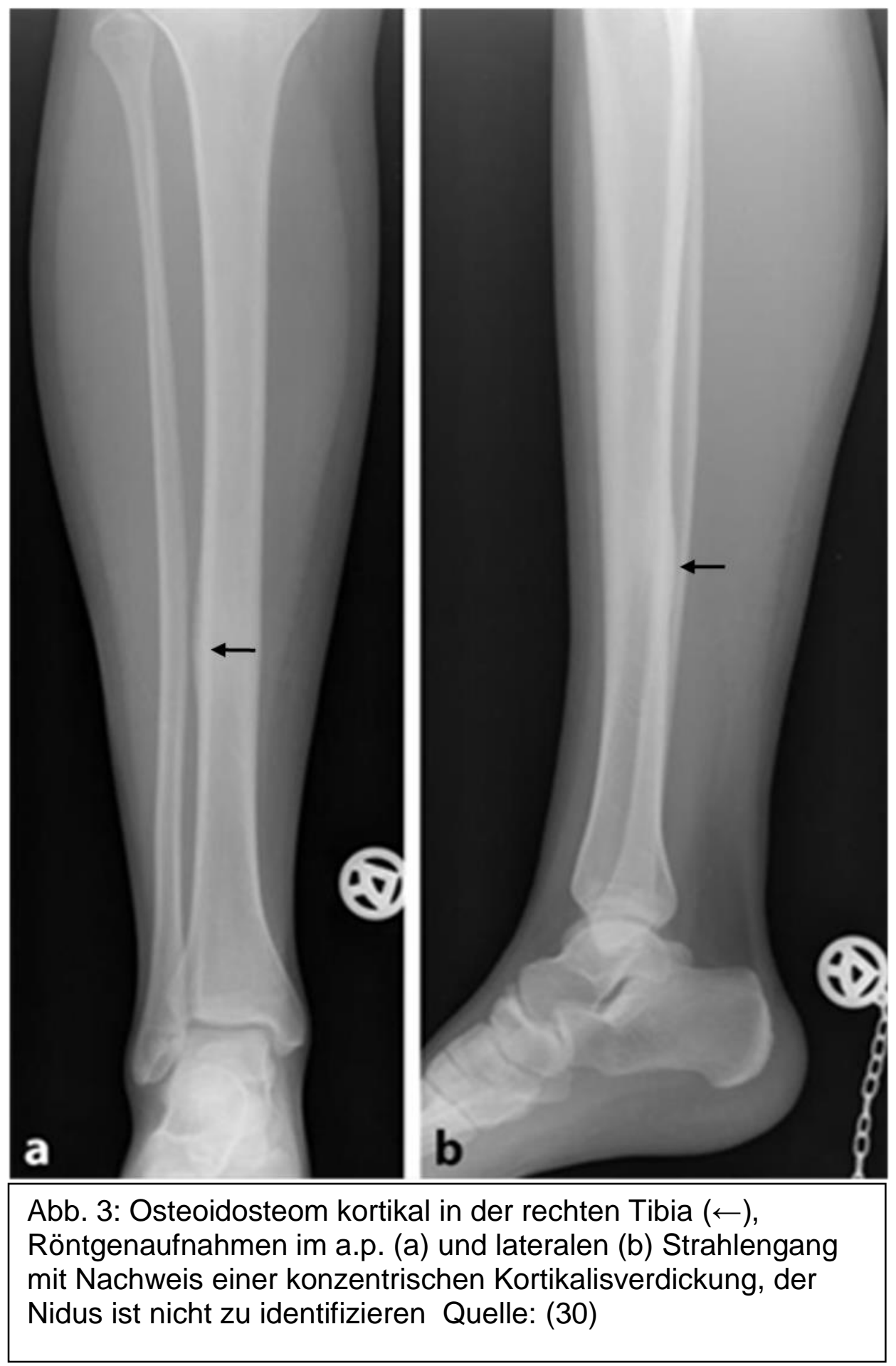

Skelettszintigraphie:

Mit intravenös verabreichten, radioaktiv markierten Substraten (so genannten Tracern, z.B. ${ }^{99 m}$ Tc-MDP) können stoffwechselaktive Regionen abgebildet werden (Abb. 4) (30). Aufgrund der deutlichen Traceranreicherung im stoffwechselaktiven Nidus, ermöglicht das Skelettszintigramm bereits im frühen Stadium die Lokalisation des $\mathrm{OO}$ und dient als unterstützendes Verfahren bei unauffälligem bzw. unklarem 
Röntgenbefund. Das für das OO pathognomonische Double-Density-Sign entsteht durch die deutliche Mehrspeicherung im hypervaskularisierten Nidus sowie die im Vergleich zum Nidus etwas geringere Tracermehrspeicherung in der umgebenden Sklerosezone (31).

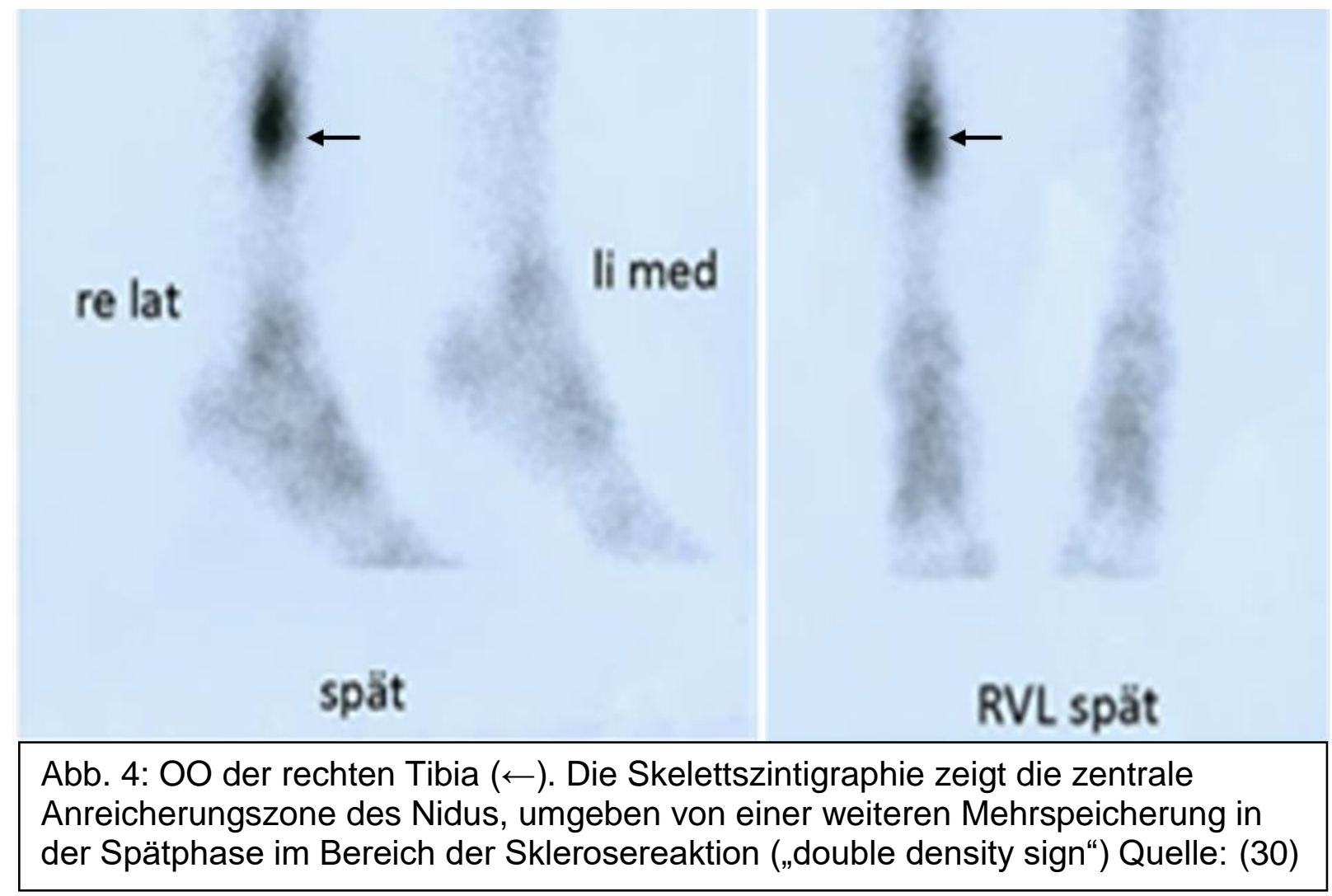

\section{Computertomographie:}

Die Dünnschicht-CT ist das bildgebende Verfahren der Wahl. Sie dient zur Sicherung der Verdachtsdiagnose und einer exakten, überlagerungsfreien Abbildung auch sehr kleiner Tumoren, unter anderem in überlagerungsreichen Körperregionen wie z.B. der Wirbelsäule. Im CT sind die typischen Zeichen des OO, Nidus mit umgebender Verknöcherung, gut nachweisbar (Abb. 5). OO-typische, zarte hypodense Linien, die zum Nidus ziehen, stellen Gefäße dar und werden als „vessel sign“ oder „vascular groove sign" bezeichnet. Die Schichtdicke und das FOV sollten möglichst klein gewählt werden, um auch $\mathrm{O}$ von geringer Größe zu detektieren. Eine Schichtdicke von $2 \mathrm{~mm}$ ermöglicht dabei die sichere Darstellung (12, 32, 33). 


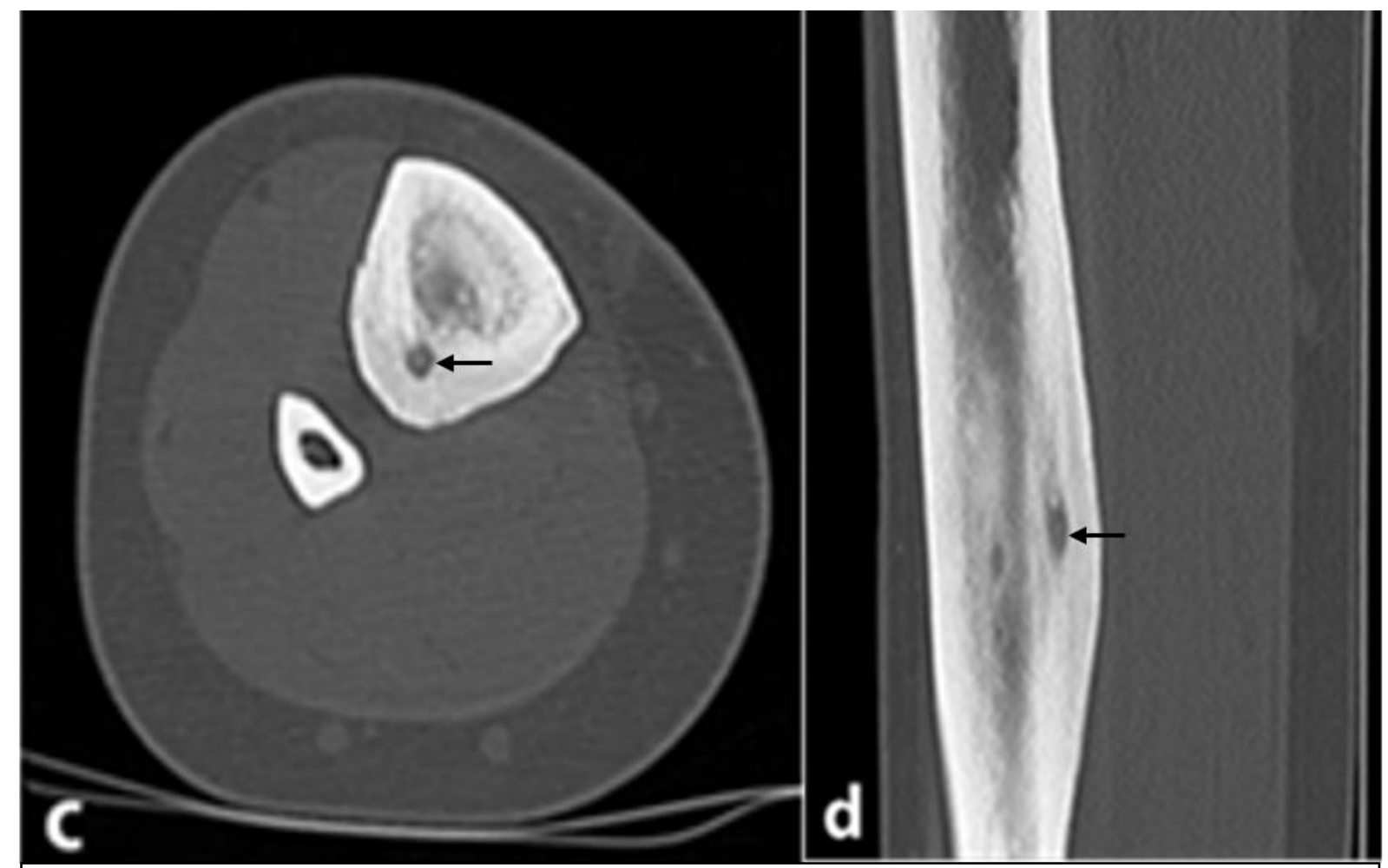

Abb. 5: OO der rechten Tibia. CT in Dünnschichttechnik in transversaler (c) und sagittaler (d) Schnittebene mit Nachweis des Nidus $(\leftarrow)$ Quelle: (30)

\section{Magnetresonanztomographie:}

Die MRT stellt eine ergänzende diagnostische Methode zur Darstellung und Lokalisation des $\mathrm{OO}$ dar (Abb. 6). Kernspintomographisch können insbesondere Weichteile und wasserreiche Strukturen mit überragendem Kontrast abgebildet werden. Somit können Knochenmarködeme oder Weichteilreaktionen z.B. im Rahmen einer Periostitis oder Synovitis, hochsensitiv detektiert werden. Hingegen kann die kalkreiche und wasserarme Knochenmatrix im Gegensatz zum CT nur eingeschränkt beurteilt werden. Daher kann die alleinige MRT-Beurteilung zu Fehldiagnosen führen, wie z.B. zur Verwechslung von Osteoidosteom und Osteosarkom (29). Zur Erleichterung der OO-Diagnose kann gadoliniumhaltiges Kontrastmittel verwendet werden. Ähnlich zur Szintigraphie zeigt sich ein deutliches Enhancement im vaskularisierten Nidus. Im Vergleich zum Dünnschicht-CT wird eine zum Teil bessere Darstellung des Nidus erreicht (34). Durch das vielfältige Erscheinungsbild des OO im MRT sollte der diagnostische Befund immer in Kombination mit einer anderen Bildgebung, z. B. CT, erfolgen, um Fehldiagnosen zu vermeiden (35). 

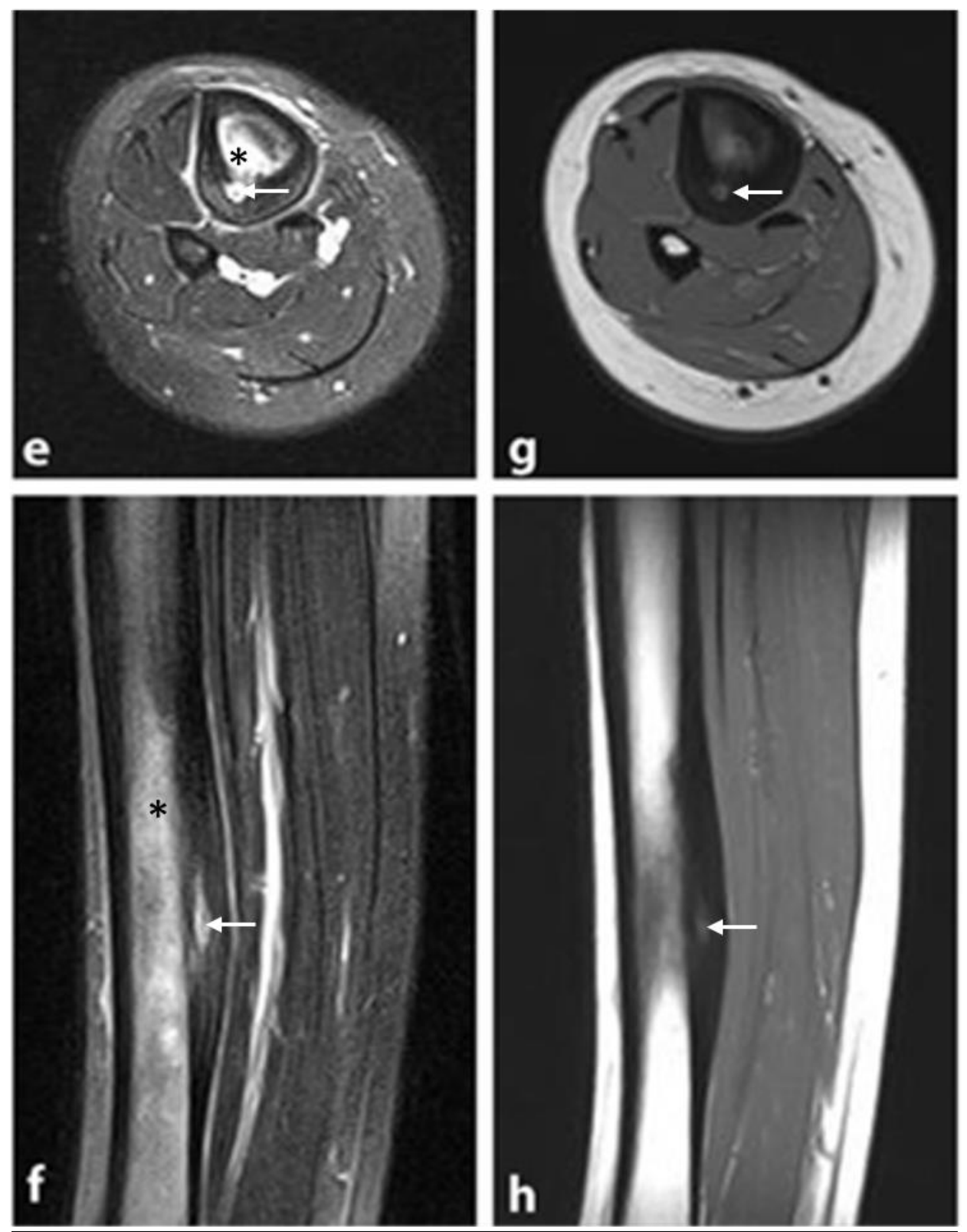

Abb. 6: OO der rechten Tibia. Im MRT stellt sich der Nidus $(\leftarrow)$ und das Knochenmarködem $\left(^{*}\right)$ auf fettgesättigten T2gewichteten Bildern signalreich $(e, f)$ dar, in der T1-Wichtung signalarmer Nidus (g, h) Quelle: (30)

\subsection{Therapie}

Ziel ist es, den Nidus vollständig zu entfernen und den Patienten dadurch von seinen Schmerzen zu befreien. Reste des Nidus können zu einem Rezidiv führen. Die Entfernung der Sklerose ist dagegen nicht nötig (12). Eine zu großzügige operative Entfernung der Tumorregion birgt wiederum das Risiko einer Instabilität des 
Knochens. Deshalb sollte der Defekt auf den Nidus begrenzt bleiben. Bildgesteuerte perkutane Ablationstechniken wurden entwickelt, um das lokale Gewebetrauma gering zu halten und so das Risiko einer Knocheninstabilität zu minimieren. Des Weiteren resultieren durch den minimalinvasiven Charakter niedrigere Komplikationsraten mit kürzere Krankenhausaufenthalte und dadurch potentiell auch geringere Kosten.

Mögliche Therapiemethoden werden im Folgenden kurz vorgestellt.

\subsubsection{Medikamentöse Schmerztherapie}

Diese etablierte medikamentöse Behandlung des OOs erfolgt mit NSAR. Diese dient sowohl als wichtiger diagnostischer Test als auch als effiziente medikamentöse Basistherapie. Eine NSAR-Dauertherapie kann in Erwägung gezogen werden, da allein die starken Schmerzen zur Beeinträchtigung der Lebensqualität führen. Unter alleiniger medikamentöser Therapie kam es in einigen Fällen zur Spontanheilung, so dass dies eine Alternative zu den operativen Verfahren sein kann. Jedoch sind die Nebenwirkungen wie z. B. Übelkeit, Schwindel und Magengeschwüre, durch die Einnahme von NSAR nicht zu unterschätzen (36). Viele Patienten ziehen einen kurativen operativen Eingriff einer symptomatischen Dauermedikation vor. Die medikamentöse Dauertherapie des OO sollte erst dann indiziert werden, wenn z.B. die körperliche Verfassung des Patienten oder die Tumorlokalisation gegen eine Intervention bzw. OP sprechen oder der Patient diese ablehnt (36). Eine schwere Nierenfunktionsstörung stellt eine Kontraindikation der medikamentösen Dauertherapie dar (37).

\subsubsection{En-bloc-Resektion}

Bei diesem offen-chirurgischen Verfahren wird nach Freipräparation des Knochens das entsprechende Knochenstück mit Hilfe von Sägen, Meißeln und Fräsern, im Ganzen - also en-bloc - entfernt. Um die Instabilität bei großen Knochendefekten zu verringern und eine schnellere Heilung zu ermöglichen, kann eine Auffüllung mit autologem Knochen oder allogenem Knochenersatz bzw. die Einbringung von Osteosynthesematerial erfolgen (38). Solange sich der Patient noch in Narkose befindet, kann eine Röntgendarstellung des Resektates erfolgen, um die komplette Entfernung des Nidus nachzuweisen. Beim Verdacht auf inkomplette Resektion des 
Nidus erfolgt eine Nachresektion. Durch den Umfang der Gewebeentfernung ist eine vollständige histopathologische Untersuchung und damit eine klare Dignitätsbestimmung mit sicherer Diagnose möglich (38). Allerdings bedingt das relevante Gewebstrauma ein erhöhtes Risiko einer Wundinfektion oder einer Osteomyelitis. Zur Reduktion des Resektionsausmaßes gibt es verschiedene Ansätze, den Nidus intraoperativ zu visualisieren wie z.B. durch präoperative CTgesteuerte Markierung, intraoperatives CT oder Gammasonde nach vorheriger Tracergabe, z.B. ${ }^{99 m T C-M D P ~(39-41) . ~}$

\subsubsection{Offene Kürettage}

Wie bei der En-bloc-Resektion erfolgt dieses Verfahren ebenfalls zumeist in Vollnarkose. Die intraoperative Visualisierung des Nidus kann mit den zuvor beschriebenen Methoden erfolgen. Nach Abtragung des Knochendeckels erfolgt das vollständige Herausschälen (Kürettage) des Nidus mit einem scharfen Löffel. Im Anschluss an eine Glättung des Knochens kann der Defekt mit dem zuvor gewonnenen Perioststück aufgefüllt werden. Somit bleibt die Stabilität im OP-Gebiet meist erhalten (13). Bei unvollständiger Entfernung des Nidus ist mit einer hohen Rezidivgefahr zu rechnen. Von Nachteil ist das fragmentierte Material, aus dem der Pathologe keine Aussage über die vollständige Entfernung des Nidus treffen kann. Auch das Operationstrauma und die relativ lange Rehabilitationszeit sind als nachteilig zu nennen. Obgleich das Ausmaß der Invasivität zur En-bloc-Resektion geringer ist, muss auf die Vollbelastung bei Eingriffen an den unteren Extremitäten für einige Wochen verzichtet werden (13).

\subsubsection{Bildgesteuerte minimal-invasive perkutane Verfahren}

Die minimal-invasive Technik wurde erstmals 1989 von Doyle und King beschrieben (42). Seitdem wird sie bei verschiedenen Therapieoptionen, z.B. der CT-gesteuerten Nidusbohrung, Alkoholinjektion oder Thermoablation, angewandt.

Mit Hilfe der CT kann der Nidus intraoperativ exakt lokalisiert werden um diesen dann bildgestützt perkutan zu sondieren. Der zu behandelnde Knochenabschnitt muss also nicht mehr freipräpariert werden. Aufgrund der geringen Invasivität sind diese Verfahren komplikationsärmer und gewebeschonender im Vergleich zu dem offen chirurgischen Vorgehen. Wegen der kürzeren Rekonvaleszenzzeit und Hospitalisation sind diese Verfahren zudem potentiell kostengünstiger. 
Perkutane Nidusdestruktion durch CT-kontrollierte Ausbohrung:

Nach einer kleinen Stichinzision der Haut wird unter CT-Kontrolle mit einem Hohlbohrer der Nidus aufgebohrt bzw. destruiert $(19,29)$. Durch die Bildsteuerung wird die korrekte Lage des Bohrers gesichert. Die Schwierigkeit besteht darin, den Nidus vollständig mit dem Bohrer zu erfassen, um Rezidive zu vermeiden. Die Hautwunde wird nach dem Eingriff mit Steristrips verschlossen und mit einem sterilen Verband versorgt. Dieses Verfahren kann in einer ambulanten Behandlung erfolgen. Zur besseren Wundheilung wird für einige Tage eine Teilbelastung empfohlen (19). Das gewonnene Material wird histopathologisch begutachtet, jedoch führt die Aufarbeitung oft nicht zur Bestätigung eines OO, da die typische Gewebestruktur zerstört wurde. Malignität kann allerdings meist ausgeschlossen werden, so dass die pathohistologische Untersuchung, v.a. bei uneindeutigen Befunden, trotzdem immer sinnvoll ist (43). Durch diese Methode konnten die Morbidität und das Ausmaß der Wunden im Vergleich zu den chirurgischen Verfahren erheblich gesenkt werden (12).

Perkutane Nidusdestruktion durch CT-kontrollierte Ausbohrung und Alkoholinjektion: Der Zugangsweg wird bei dieser Methode wie oben beschrieben etabliert. Nach der Ausbohrung des Nidus wird der Bohrer entfernt und über eine dann eingebrachte Nadel 0,5 - 1,0 ml Ethanol (96\% Alkohol) injiziert. Dadurch wird eine zusätzliche chemische Destruktion des Nidus bewirkt. Bei OO in Angrenzung an neurovaskuläre Strukturen oder die Wachstumsfugen sollte aufgrund der Nekrosegefahr auf die Ethanolinjektion verzichtet werden. Durch die zusätzliche Nidusdestruktion durch das applizierte Ethanol können kleinere Bohrer im Vergleich zur alleinigen Ausbohrung verwendet werden. Dies verringert das Operationstrauma weiter und die sich anschließende körperliche Belastbarkeit ist schneller wiederhergestellt (44). Bei größeren Nidi sollten mehrere Zugangswege gewählt werden, um die Verteilung des Ethanol zu verbessern (44). Bei der Injektion von mehr als $1 \mathrm{ml}$ Ethanol steigt das Risiko von schweren Nekrosen in den angrenzenden Weichteilen (45). Nachteilig ist, dass sich die Verteilung des injizierten flüssigen Alkohols im Zielgebiet schlecht kontrollieren lässt.

Perkutane Nidusdestruktion durch laserinduzierte Thermoablation:

Nach erfolgter Anästhesie und kleiner kutaner Stichinzision erfolgt die CT-geführte Platzierung der Ablationssonde in das OO. Bei starker Sklerosierung kann die 
Etablierung des Zugangsweges durch einen Bohrer ermöglicht werden. Die CT dient zur Überprüfung der Lage der Sonde im Zentrum des Nidus. Die laserinduzierte Thermoablation folgt je nach Systemhersteller anhand dedizierter Protokolle (z.B. $90^{\circ} \mathrm{C}$ für 10 Minuten). Bei einem großen Nidusdurchmesser (>10 mm) muss die Ablation zum Teil über verschiedene Zugangswege durchgeführt werden (46, 47). Ziel ist die Devaskularisation des Nidus sowie die Destruktion von Nervenfasern und prostaglandinproduzierenden Zellen, was die Schmerzfreiheit des Patienten bewirken soll. Im Gegensatz zu onkologischen Thermotherapien muss beim $\mathrm{OO}$ das umgebende Gewebe (Sklerosesaum) nicht abgetötet werden. Deshalb können niedrigere Energien zur Ablation verwendet werden, was zur Gewebeschonung beiträgt. Das geringe Operationstrauma des Knochens mit erhaltener Belastbarkeit ist ein deutlicher Vorteil $(46,47)$. Wenn Bohrsysteme Verwendung finden, kann auch Material für eine histopathologische Untersuchung gewonnen werden. Durch die Hitzeentwicklung kann es zur Schädigung neurovaskulärer Strukturen kommen, weshalb sensible Strukturen mindestens $10 \mathrm{~mm}$ entfernt von der Nadelspitze liegen sollten (46).

\section{Perkutane Nidusdestruktion durch Radiofrequenzablation (RFA):}

Erstmalig wurde die RFA zur Therapie des OO 1992 von Rosenthal beschrieben (48). Hierbei erfolgt ebenfalls unter Vollnarkose und nach kutaner Stichinzision die CT-geführte Platzierung der Ablationsnadel. Vorerst wird der Zugang durch den Knochen in den Nidus perkutan durch einen Bohrer etabliert. Das gewonnene Gewebematerial zur histopathologischen Untersuchung ist aufgrund der geringen Menge und der Fragmentierung leider häufig nur eingeschränkt beurteilbar. Nach Platzierung der Thermoablationsnadel im Nidus wird ein hochfrequenter elektrischer Wechselstrom $(375-480 \mathrm{kHz})$ über den nicht isolierten Anteil einer Thermosonde verabreicht. Durch die induzierten Temperaturen von $90-100^{\circ} \mathrm{C}$ erfolgt die thermische Destruktion des Nidus (49). Auch hier folgt die Ablation herstellertypischen Ablationsprotokollen, die in der Regel nur wenige Minuten dauern. Zur peri- und postinterventionellen Schmerztherapie kann ein Lokalanästhetikum (z.B. Xylocain) verwendet werden. Auch bei dieser Methode sind bei großen Nidi mehrere Ablationszyklen mit umpositionierter Nadel nötig, um die komplette Ablation des Nidus zu gewährleisten $(19,50)$. Wie bei der zuvor beschriebenen Ablationstechnik ist auch hier nur ein schmaler knöcherner Zugang nötig, welcher die 
Knochenstabilität nicht beeinträchtigt und die sofortige Belastung ermöglicht (50). Bei Rezidiven ist eine erneute Ablation möglich. Als Komplikationen sind Hautverbrennungen am häufigsten, v.a. bei $\mathrm{OO}$ mit kleinem Abstand zur Kutis. Zu wichtigen neurovaskulären Strukturen sollte ein Sicherheitsabstand von ca. $1 \mathrm{~cm}$ eingehalten werden (51). OO in der Wirbelsäule oder im Handbereich können in Einzelfällen aufgrund ihrer Lage eine Kontraindikation für die RFA sein $(19,50)$.

Bei der Auswahl des Therapieverfahrens muss neben der lokalen Verfügbarkeit natürlich auch der Wunsch des Patienten Berücksichtigung finden. Die initiale genaue bildgebende Lokalisation des Nidus sowie angrenzender Strukturen sind sehr wichtig um die Behandlung genau zu planen und dem Patienten das optimale Therapieverfahren anzubieten. Angrenzende sensible Strukturen wie z.B. die Epiphysenfuge, Gelenkknorpel, Haut, Rückenmark sowie Gefäße und Nerven müssen in jedem Fall geschont werden. Deshalb kann es in manchen Fällen sinnvoll sein den Nidus nur durch Ausbohrung zu behandeln und auf die zusätzliche alkoholische oder thermische Destruktion zu verzichten. Bei kleinen Nidi sollten, wie bereits erwähnt, auch kleinere Bohrsysteme Verwendung finden um das Operationstrauma weiter zu minimieren. Eigene Erfahrungen haben gezeigt, dass bei der Behandlung kleiner Knochen besonders vorsichtig agiert werden muss. Hier ist das Verhältnis zwischen der Größe des Knochens und dem gesetzten Trauma relativ klein, so dass es vermehrt zu einer posttherapeutischen Instabilität bzw. Fraktur kommen kann.

Insgesamt wird die CT-gesteuerte RFA mittlerweile von vielen Anwendern als die Standardtherapie des $\mathrm{OO}$ angesehen. 
5. Publikationsmanuskript

Analyse der Lebensqualität von Patienten mit Osteoidosteom vor und nach Behandlung mittels Radiofrequenzablation

\section{Seider, M. Reinhardt, D. Zajonz, P. Voigt, T. Kahn \& M. Moche}

Der Chirurg

Zeitschrift für alle Gebiete der operativen Medizin

ISSN 0009-4722

Chirurg

DOI 10.1007/s00104-019-0818-3

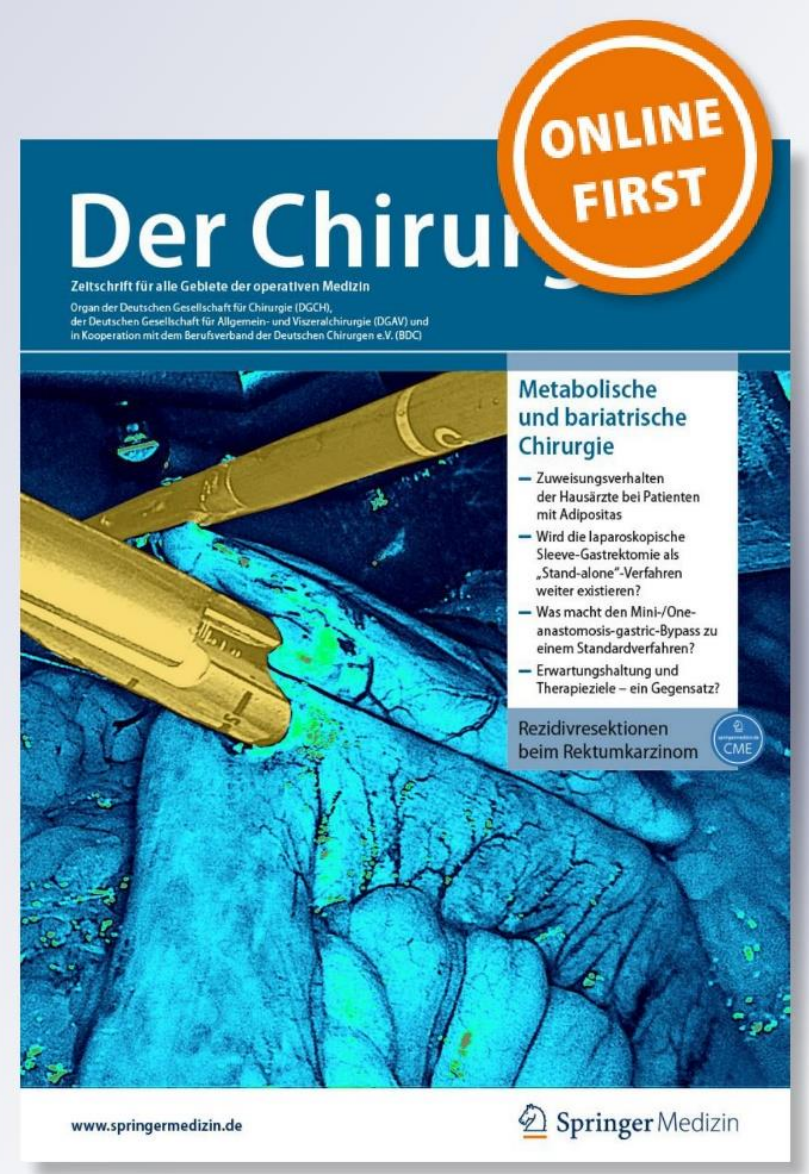

算 Springer 


\section{Der Chirurg}

\section{Originalien}

Chirurg

https://doi.org/10.1007/s00104-019-0818-3

(c) Springer Medizin Verlag $\mathrm{GmbH}$, ein Teil von Springer Nature 2019

\section{Seider ${ }^{1} \cdot$ M. Reinhardt ${ }^{1} \cdot$ D. Zajonz ${ }^{2} \cdot$ P. Voigt ${ }^{1} \cdot$ T. Kahn ${ }^{1} \cdot$ M. Moche}

Medizinische Fakultät der Universität Leipzig, Klinik und Poliklinik für Diagnostische und Interventionelle Radiologie, Universitätsklinikum Leipzig AöR, Leipzig, Deutschland

${ }^{2}$ Klinik und Poliklinik für Orthopädie, Unfallchirurgie und Plastische Chirurgie, Universitätsklinikum Leipzig AöR, Leipzig, Deutschland

\section{Analyse der Lebensqualität von Patienten mit Osteoidosteom vor und nach Behandlung mittels Radiofrequenzablation}

\section{Einleitung}

Das Osteoidosteom (OO) als eigene Entität wurde erstmalig 1935 von Jaffe beschrieben [7]. Dieser primäre, langsam wachsende Knochentumor macht ca. $10 \%$ der gutartigen Knochentumoren aus [9]. Etwa $80 \%$ der Patienten sind zwischen 5 und 24 Jahren alt, mit einer Verteilung männlich zu weiblich von 3:1. Ca. $50 \%$ aller OO sind in Femur und Tibia lokalisiert, am häufigsten tritt es im proximalen Oberschenkel auf [3]. $10 \%$ aller OO treten in der Wirbelsäule auf [18].

Die klinischen Symptome sind charakteristisch mit starken, vor allem nachts auftretenden Schmerzen, die auf nichtsteroidale Antiphlogistika ansprechen $[9,16]$. Die medikamentöse Langzeittherapie ist aufgrund der bekannten Nebenwirkungen nicht unproblematisch [12].

Verschiedene bildgebende Methoden stehen zur Diagnostik des OO zur Verfügung. Die Computertomographie (CT) besitzt die höchste Genauigkeit in der Detektion des Nidus und somit des OO [1]. In der CT und im nativen Projektionsradiogramm findet man einen strahlendurchlässigen, manchmal kalzifizierten zentralen Nidus mit umgebender Knochensklerose und einer Periostverdickung. Auch die Magnetresonanztomographie (MRT) und die Knochenszintigraphie können zur Diagnosesicherung beitragen wobei die In-

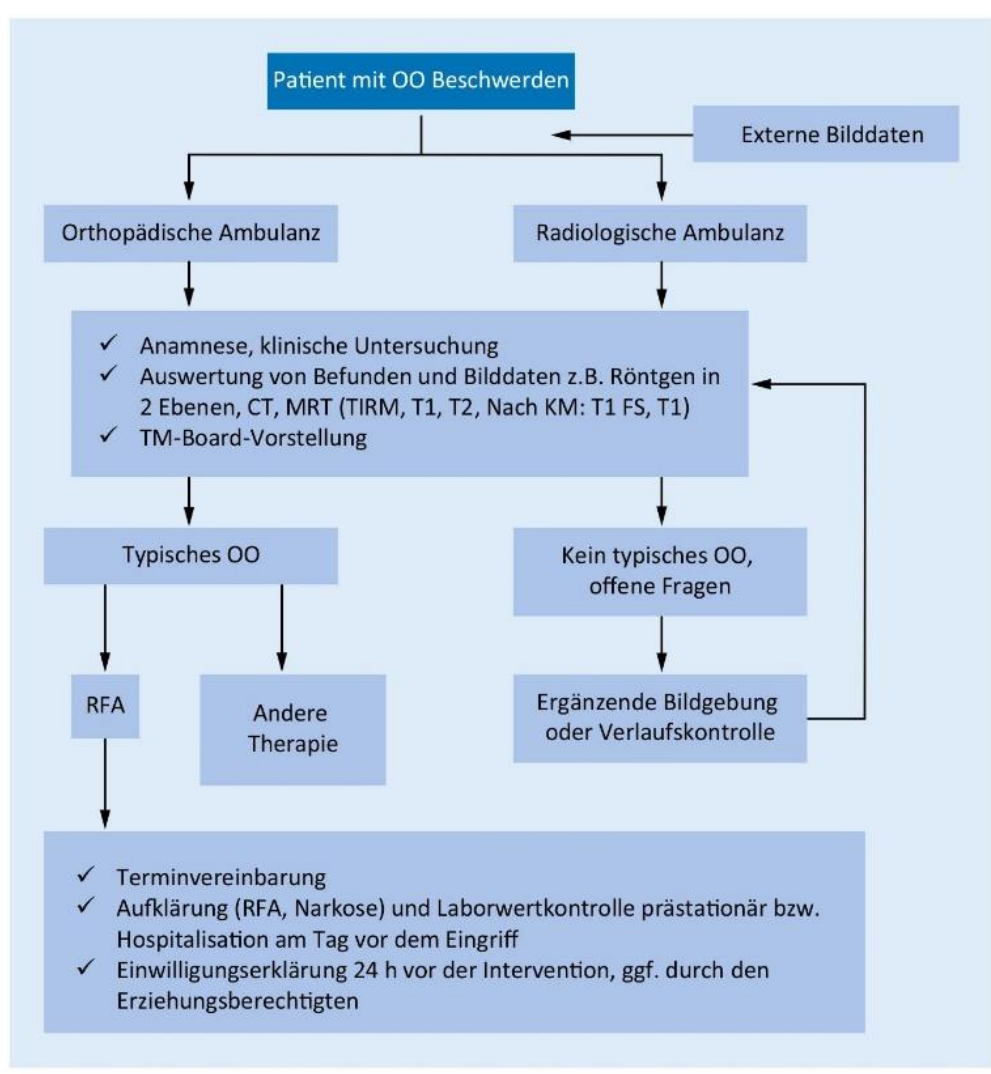

Abb. 1 ^ Präinterventionelles Workflow-Schema. CTComputertomographie, KM Kontrastmittel, MRT Magnetresonanztomographie (Sequenzen in unterschiedlichen Wichtungen), 00 Osteoidosteomen, RFA Radiofrequenzablation, TM-Board Tumorboard 


\section{Author's personal copy}

\section{Originalien}

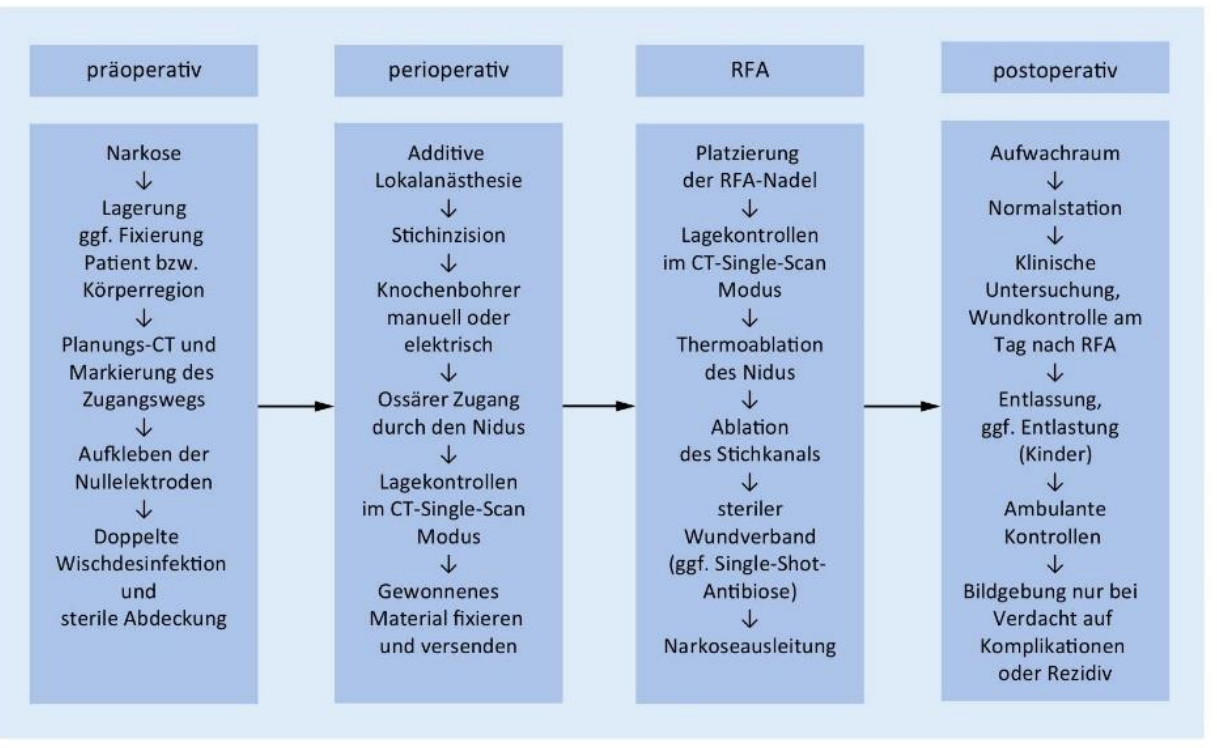

Abb. $2<$ Periinterventionelles Workflow-Schema. CT Computertomographie, RFA Radiofrequenzablation terpretation immer im Zusammenhang mit dem Projektionsradiogramm und CT erfolgen sollte $[1,5,16]$. Die chirurgische Exzision galt lange als "Goldstandard“ zur Behandlung des OO. 1992 schließlich berichteten Rosenthal et al. über die ersten perkutanen thermalen Ablationen beim OO [13]. Seitdem wurden viele Studien veröffentlicht, in denen die Effektivität insbesondere CT-gestützter Radiofrequenzablationen belegt wurde $[3,8,9,16,18]$. Dies belegen primäre Erfolgsraten von $56-100 \%$ bzw. $89-100 \%$ bei wiederholter Anwendung [14]. Der klinische Erfolg ist mit der chirurgischen Intervention vergleichbar, bei jedoch deutlich kürzerer Hospitalisation [14] und geringeren Komplikationsraten [17]. Insbesondere $\mathrm{OO}$ mit Gelenkbeteiligung bzw. intraartikuläre $\mathrm{OO}$ sollten frühzeitig diagnostiziert und behandelt werden, um schwerwiegende Folgeschäden im Gelenk zu vermeiden. Die Behandlung mit Chemotherapie oder gar Radiatio wird nicht empfohlen [6].

Ziel dieser Arbeit ist es, die Schmerzintensität und die davon abhängige Lebensqualität prä- und postinterventionell zu vergleichen und die histologische Aufarbeitung auszuwerten. Des Weiteren wird ein detailliertes Schema (• Abb. 1 und 2) für eine standardisierte Prozedur zu Verfügung gestellt.

\section{Methodik}

\section{Studienpatienten}

Von Juli 2004 bis Oktober 2015 wurden 26 Patienten (m:w 18:8) mit 27 Knochenläsionen mittels CT-gestützter RFA behandelt. Eine Patientin wurde aufgrund eines Rezidivs wiederholt mittels RFA behandelt. Neben der typischen klinischen Symptomatik dienten bei allen Patienten Röntgenaufnahmen und eine dünnschichtige native CT der Tumorregion zur Diagnosesicherung. 11 Patienten hatten im Rahmen einer extern durchgeführten Diagnostik schon eine MRT mit Kontrastmittel erhalten, bei der ein Herd mit deutlichem Kontrastmittel-Enhancement darstellbar war.

\section{Behandlung}

Jeder Patient mit klinisch und bildmorphologisch gesichertem OO wurde im interdisziplinären Board für Knochentumoren vorgestellt. Im Konsens wurde die Entscheidung zur Behandlung mittels RFA getroffen (- Abb. 1). Im Regelfall erfolgte die stationäre Aufnahme der Patienten am Tag vor der RFA, u.a. um anhand der Blutwerte (Thrombozyten, Quick-Wert) einen suffizienten Gerinnungsstatus sicherzustellen. $24 \mathrm{~h}$ vor der
Prozedur erfolgte die ausführliche Aufklärung.

Die Ablation erfolgte in Allgemeinanästhesie und additiver lokaler Anästhesie des Periosts mit 1-2 $\mathrm{ml} 1 \%$ igem Xylokain sowie unter streng sterilen Kautelen. Nach Anfertigung eines Planungs-CT wird über eine ca. $1 \mathrm{~cm}$ breite Stichinzision mit einem Bohrsystem der ossäre Zugangsweg etabliert. Dazu wurde die manuelle Knochenmarkbiopsiekanüle $(\mathrm{O}-$ MaxTM $11 \mathrm{G} \times 10 \mathrm{~cm}$ HVM Medical Products GmbH, Rotenburg, Deutschland) oder das elektrische Bohrsystems (11 G $\times 102$ mm ARROW ${ }^{\circledR}$ OnControl ${ }^{\circledast}$ Bone Marrow Biopsy System Tray and Power Driver, Vidacare ${ }^{\circledast}$, Teleflex Incorporated, Wayne, PA, USA) verwendet. Ziel ist es, die Kortikalis möglichst im $90^{\circ}$-Winkel zu treffen, um ein Abrutschen des Bohrers zu vermeiden. Jedoch wurde auch darauf geachtet, neurovaskuläre Strukturen zu schonen und einen transartikulären Zugang zu vermeiden. Dazu war es zum Teil nötig, den Nadelverlauf etwas anguliert zu planen ( $\mathbf{A} \mathbf{A b}$. 3) oder den Zugang durch die intakte Kortikalis der Gegenseite zu wählen (๑ Abb. 4). Bei 22 Patienten wurde gewonnenes Material in $4 \%$ iger Formalinlösung fixiert und anschlieBend histologisch untersucht. Danach wird die RFA-Nadel im Nidus platziert und die Thermoablation nach einem 


\section{Author's personal copy}

\section{Zusammenfassung · Abstract}

standardisierten, impedanzgesteuerten Protokoll durchgeführt. Zur Ablation nutzen wir die Soloist" Single Needle Electrode $(16,5 \mathrm{G} \times 18 \mathrm{~cm}$, Aktivelektrode $0,9 \mathrm{~cm}$ lang, Boston Scientific, Marlborough, MA, USA) und den RFGenerator RF 3000" der Firma Boston Scientific. Bei einem Patienten mit einer Nidusgröße von $15 \mathrm{~mm}$ wurde das LeVeen $^{\text {sx }}$ SuperSlim ${ }^{\text {*x }}$ Needle Electrod System $(2,0 \times 15 \mathrm{~cm}$, Boston Scientific, Marlborough, MA, USA) verwendet, wobei abweichend vom Standardprotokoll 3 überlappende RF-Zyklen mit nur $1 \mathrm{~cm}$ ausgefahrener Schirmchenelektrode und einer Leistung von bis zu $30 \mathrm{~W}$ bis zum Roll-Off ${ }^{\circledR}$ (jeweils ca. $2 \mathrm{~min}$ ) erfolgten ( $\square$ Abb. 5). Die Platzierung von Bohrer und Nadel erfolgt standardmäßig CT-gestützt im Single-ScanModus. Bei einem Patienten erfolgte bei der Behandlung eines $\mathrm{OO}$ an der ventralen Wirbelkörperkante in Höhe des Brustwirbelkörpers (BWK) 12 über eine CHIBA-Biopsie-Feinnadel $(22 \mathrm{G}, 20 \mathrm{~cm}$, Peter Pflugbeil GmbH, Medizinische Instrumente, Zorneding, Deutschland) die Luftinsufflation, um eine Luftdissektion der Aorta zu erreichen ( $\square$ Abb. 6). Bei jungen Patienten (geringes Körpergewicht) bzw. einer Lokalisation des OO im Bereich der Extremitäten erfolgt die Fixierung teilweise in einer Vakuummatratze, um Lageänderungen nach der Planungsbildgebung und während des eigentlichen Eingriffs zu verhindern (- Abb. 3).

Abschließend wird der Stichkanal im Rückzug abladiert und der kutane $\mathrm{Zu}$ gangsweg mit einem sterilen Verband versorgt. Im Aufwachraum wird der Patient nach der Prozedur für einige Stunden überwacht. Wenn keine Beschwerden vorliegen, wird der Patient am darauffolgenden Tag entlassen. Wundkontrollen erfolgen im Rahmen der ambulanten Nachsorge ( $\mathbf{\square}$ Abb. 2).

\section{Studienprotokoll}

Zur prospektiven Erfassung des Outcomes, der Schmerzintensität und der Lebensqualität wurde ein Fragebogen erstellt. Neben allgemeinen Fragen zur Schmerzart und -häufigkeit wurde u.a. eruiert, in welchen zeitlichen Abständen

Chirurg https://doi.org/10.1007/s00104-019-0818-3

(c) Springer Medizin Verlag GmbH, ein Teil von Springer Nature 2019

D. Seider · M. Reinhardt · D. Zajonz · P. Voigt $\cdot$ T. Kahn $\cdot$ M. Moche

Analyse der Lebensqualität von Patienten mit Osteoidosteom vor und nach Behandlung mittels Radiofrequenzablation

Zusammenfassung

Hintergrund. Ziel dieser Arbeit ist es, die Effektivität der Behandlung von Osteoidosteomen (00) mittels Radiofrequenzablation (RFA) zu untersuchen. Dabei werden insbesondere die Rezidivrate und die Verbesserung der Lebensqualität durch die Reduktion der Schmerzintensität bewertet.

Methodik. Retrospektive Analyse von

26 Patienten nach RFA eines 00 und prospektive Analyse von 14 Patienten mittels eines selbstentwickelten Fragebogens zur Lebensqualität. Es erfolgte die Aufarbeitung des Fragebogens, der elektronischen Patientenakte und der histopathologischen Befunde.

Ergebnisse. Durchschnittlich vergangen 22 Monate zwischen erstem Auftreten von

Analysis of the quality of life of patients with osteoid osteoma before and after radiofrequency ablation treatment

Abstract

Background. The aim of this study was to investigate the effectiveness of the treatment of osteoid osteomas $(\mathrm{OO})$ by radiofrequency ablation (RFA). In particular, the recurrence rate and the improvement in the quality of life as assessed by the reduction of pain intensity were evaluated.

Methods. Retrospective analysis of 26 patients after RFA of an $\mathrm{OO}$ and prospective analysis of 14 patients using a self-developed quality of life questionnaire. The ques tionnaire, the electronic patient file and the histopathological findings were processed. Results. An average of 22 months passed between the first onset of complaints and the
Beschwerden und der ursächlichen Therapie mittels RFA. Nach der RFA zeigte sich eine signifikante Reduktion der Schmerzsymptomatik und damit eine Steigerung der Lebensqualität. Unsere Ergebnisse bestätigen dass die RFA von $\mathrm{OO}$ ein sicheres und effizientes Therapieverfahren ist.

Diskussion. Um lange konservative Therapieversuche zu vermeiden und Auswirkungen auf das muskuloskeletale System zu vermindern, sollte nach Diagnosestellung eine zeitnahe RFA des $\mathrm{OO}$ erfolgen.

Schlüsselwörter

Knochentumor - Osteoidosteom · Minimalinvasive Therapie - Radiofrequenzablation causative treatment by RFA. After RFA, there was a significant reduction in pain symptoms and thus an increase in the quality of life. These results confirm that RFA from 00 is a safe and efficient treatment procedure. Discussion. In order to avoid long-term conservative treatment attempts and to reduce effects on the musculoskeletal system, a timely RFA of $\mathrm{OO}$ should be performed afte diagnosis.

Keywords

Bone tumor - Osteoid osteoma - Minimally invasive treatment $\cdot$ Radiofrequency ablation
Arztbesuche erfolgten, die RFA durchgeführt wurde und welche alternativen Therapieverfahren angewandt wurden. Des Weiteren wurde die Art der durchgeführten Bildgebungen erfragt. Ein wesentlicher Bestandteil des Fragebogens war die Dokumentation der Beeinträchtigung der Lebensqualität im Verlauf vom ersten Schmerzereignis sowie vor und nach der RFA. Die Graduierung erfolgte mittels visueller Analogskala (VAS) mit Werten zwischen 0 (keine Beeinträchtigung bzw. kein Schmerz) und
10 (maximale Beeinträchtigung bzw. Schmerzen).

In einer retrospektiven Auswertung der klinikeigenen elektronischen Patientenakte wurden die histopathologischen Befunde erfasst. Auch bei den behandelten Patienten mit OO, die nicht an der Befragung teilnahmen, wurde nach Hinweisen für ein Rezidiv gesucht.

Für die vorliegende Untersuchung liegt ein positives Votum der lokalen Ethikkommission vor (IRB-Nummer: IORG0001320/IRB0001750). 


\section{Author's personal copy}

\section{Originalien}
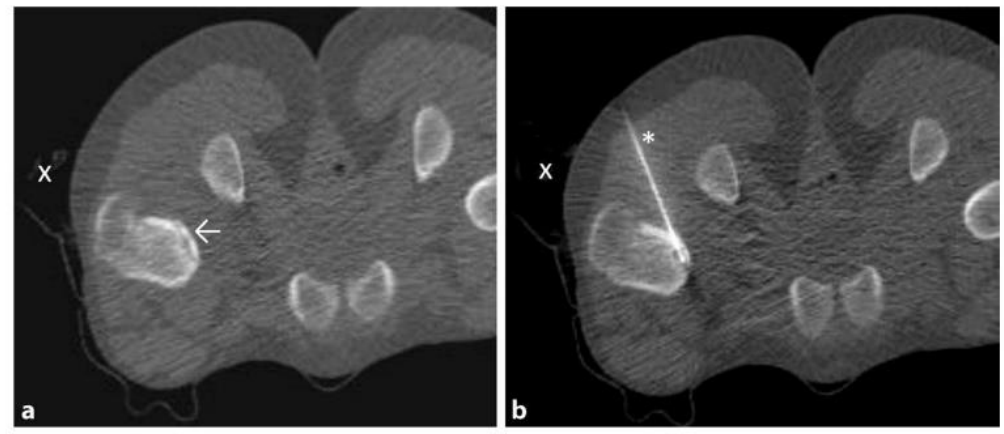

Abb. $3 \Delta$ Osteoidosteom im rechten Schenkelhals, Patient in Bauchlage. a XVakuummatratze, Pfeil Bohrkanal durch den Nidus, $\mathbf{b} \times$ Vakuummatratze, * platzierte Radiofrequenzablationselektrode
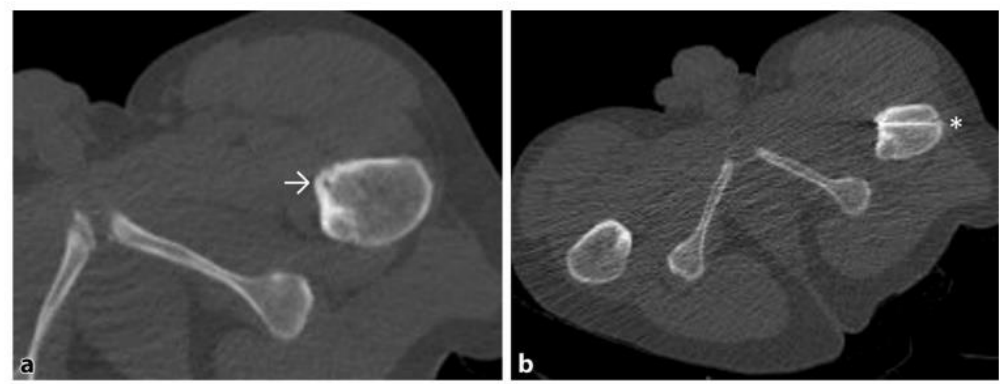

Abb. 4 \ Osteoidosteom im linken Schenkelhals, Patient in Rückenlage, links unterpolstert. a Pfeil Nidus, $\mathbf{b}$ * platzierte Radiofrequenzablationselektrode via Kortikalis der Gegenseite

\section{Statistische Analysen}

Normalverteilte Werte werden mit Mittelwerten und Standartabweichung beschrieben. Mittels Varianzanalyse (ANOVA) und dem Least-SquareMeans-Test mit Tukey-Korrektur wurden Schmerzintensitäten $(S z 1=$ initial, $\mathrm{Sz2}=$ vor RFA, Sz3 = direkt nach RFA und $\mathrm{Sz} 4=$ Follow-up) und die Lebensqualität (LQ $1=$ initial, LQ $2=$ vor RFA und LQ 4 im Follow-up) zu unterschiedlichen Zeitpunkten $(\mathrm{T} 1=$ initial, $\mathrm{T} 2=$ vor RFA, T3 = direkt nach RFA und T4= Followup) verglichen. Der Pearson-Korrelationkoeffizient diente der Bewertung von körperlicher Aktivität, Leistung und Lebensqualität in Abhängigkeit von der Schmerzintensität.

\section{Ergebnisse}

\section{Studienpatienten und Tumorpathologie}

Das Durchschnittsalter der Patienten betrug 22,8 $\pm 12,7$ Jahre (4-64 Jahre). Die
Nidusgröße lag zwischen 2 und $15 \mathrm{~mm}$ $(6 \pm 3,3 \mathrm{~mm})$, wobei eine $15 \mathrm{~mm}$ große Läsion im Grenzbereich zum GiantOsteoidosteom bzw. Osteoblastom lag. Elf Patienten zeigten die häufigste OOLokalisation im Femur. Vier OO fanden sich in der Tibia und ein OO im Azetabulum. An der oberen Extremität lagen zwei OO im Caput humeri, jeweils ein OO in der Ulna und im rechten Digitus IV. Des Weiteren zeigten sich jeweils ein $\mathrm{OO}$ im rechten Wirbelkörperbogen von BWK 11, im linken Wirbelkörperbogen von BWK 4, im rechten Pedikel von BWK 9 sowie im BWK 12 und LWK 5. Ein OO war im Os naviculare lokalisiert.

Erwähnenswert ist, dass bei einer jungen Patientin (16 Jahre) bei Verdacht auf eine rheumatische Erkrankung bereits ein Therapieversuch mit niedrigdosierektomie durchgeführt wurde, bevor die Diagnose eines OO im rechten Schenkelhals gestellt wurde.

Die Auswertung der 22 zur pathologischen Untersuchung eingesandten tem Methotrexat und operativer Synov-
Proben ergab folgende Ergebnisse: Drei Präparate enthielten keine repräsentative Materialmenge und waren damit nicht auswertbar bezüglich eines OO. In 17 Fällen (77\%) entsprach der Befund einem OO bzw. war damit vereinbar. Bei zwei Materialproben konnten histopathologisch keine typischen OOVeränderungen gefunden werden.

\section{Technische Schwierigkeiten und} Komplikationen

Insgesamt beobachteten wir bei den 27 RFA von OO drei Komplikationen. Davon zogen zwei eine additive Behandlung des Patienten nach sich.

Bei einem Patienten brach bei der Anlage der manuellen Knochenbohrung das Handstück des Bohrers, sodass dieser fest im Patienten stecken blieb. Erst unter Verwendung entsprechender Werkzeuge aus einem orthopädischen Operationssieb konnte dieser gelockert und entfernt werden. Durch Verwendung eines zweiten manuellen Bohrers konnte der Bohrkanal vollständig angelegt und die $\mathrm{Ab}$ lation erfolgreich durchgeführt werden. Diese technische Komplikation führte zu keinerlei Beeinträchtigung bei dem $\mathrm{Pa}$ tienten.

Bei einem Patienten entwickelte sich nach vollständiger Ablation des Nidus ein Weichteilabszess im Zugangsweg. Dieser heilte nach chirurgischer Spaltung und Antibiotikatherapie vollständig aus. Eine Osteitis bzw. Osteomyelitis entwickelte sich nicht.

Eine junge Patientin (• Tab. 1, Patient Nr. 8) mit einem $\mathrm{OO}$ im Os naviculare des rechten Fußes, welches im November 2014 mittels RFA behandelt wurde, stellte sich bei persistierender Beschwerdesymptomatik wiederholt in der orthopädischen Ambulanz vor. Untypisch waren die belastungsabhängigen Schmerzen (Schmerzintensitäten bis 7 in der VAS). Im Röntgen waren, abgesehen von einer Hypersklerosierung im Os naviculare, keine Auffälligkeiten am Fußskelett sichtbar. In einem erneut durchgeführten Röntgenbild im Januar 2016 demarkierte sich eine Fraktur des Os naviculare in Lokalisation des ehemaligen OO. Hier wurde der Verdacht auf eine Osteonekrose und eine pathologische Fraktur nach 


\section{Author's personal copy}

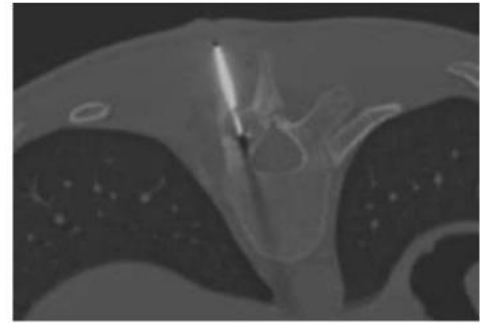

Abb. 5 A Osteoidosteom im Brustwirbelkörper 11, Wirbelbogen rechts, entfaltete Schirmchenelektrode

RFA geäußert. Leider entzog sich die Patientin dem weiteren Follow-up, sodass die Verdachtsdiagnose weder widerlegt noch bestätigt werden konnte.

\section{Outcome und Befragung}

Alle Patienten wurden prospektiv befragt und erhielten dazu einen Fragebogen per E-Mail oder Post zugesandt. Vierzehn Patienten antworteten mit einem vollständig ausgefüllten Fragebogen und gaben ihr schriftliches Einverständnis zur pseudonymisierten Verwendung ihrer Daten. Bei offenen Fragen wurden die Daten durch ein telefonisches Interview komplettiert. Die übrigen Patienten antworteten nicht bzw. konnten wegen geänderter Adressdaten nicht mehr kontaktiert werden.

Von den 26 behandelten Patienten musste eine junge Patientin 8 Monate nach der ersten RFA bei erneut aufgetretenen OO-typischen Beschwerden erneut mit RFA am bestehenden Rezidiv behandelt werden. Die Nachbeobachtungszeit betrug im Durchschnitt $512 \pm 609$ Tage (2-2040 Tage). Bei allen Behandlungen konnte ein primärer technischer Erfolg (100\%) verzeichnet werden. Der klinische Erfolg nach der ersten Behandlung betrug $96,3 \%$ und nach der zweiten Behandlung $100 \%$.

Durchschnittlich dauerte es 3 Monate, bis die befragten Patienten sich nach dem ersten Schmerzereignis, an das sie sicher erinnern konnten, beim Arzt vorstellten (0-12 Monate). Jedoch lagen die Zeiträume bis zur Behandlung mittels RFA nach dem ersten Arztbesuch deutlich darüber (22 Monate, 5-110 Monate).

In der durchgeführten Varianzanalyse für die Schmerzintensitäten zeigten
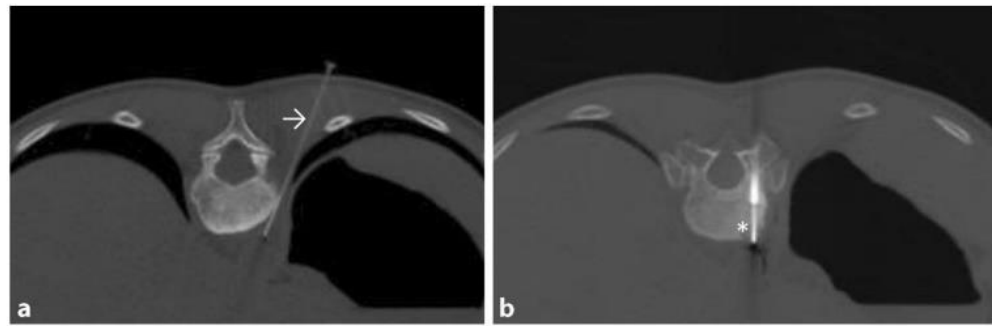

Abb. 6 ム Osteoidosteom links ventral im Brustwirbelkörper 12. a Pfeil einliegende CHIBA-Feinnade mit Luftinsufflation zum Schutz der Aorta vor der Ablationszone, $\mathbf{b}^{*}$ transpedikulär im Nidus platzierte Radiofrequenzablationssonde

sich folgende statistische Signifikanzen im zeitlichen Verlauf: Szl:Sz4 $p=<$ 0,0001, Sz1:Sz3 $p=0,0037$ und Sz2:Sz4 $p=0,0007$. Die übrigen Analysen für die Schmerzintensitäten wiesen keine Signifikanz auf, insbesondere nicht zwischen Szl und Sz2. Eine statistische Korrelation für Alter und Geschlecht haben die Statistik nicht signifikant beeinflusst.

Für die Beeinträchtigung der Lebensqualität im Vergleich zu den Zeitpunkten zeigten sich ähnliche Ergebnisse mit einer statistischen Signifikanz LQ 1:LQ 4 $p<0,0001$ und LQ 2 LQ $4 p<0,0001$ sowie nichtsignifikant LQ 1:LQ $2 p=0,9881$. Eine Trennung nach Alter und Geschlecht haben die Statistik nicht signifikant beeinflusst.

Folgende Vergleiche zeigten signifikante Korrelationen: zwischen Schmerzintensität und der Beeinträchtigung der körperlichen Aktivität $(n=14) \mathrm{r}=0,6759$ und $p=0,008$, zwischen Schmerzintensität und Beeinträchtigung der Leistung ( $n=13$, bei einem Patienten konnte keine Angabe bez. der Beeinträchtigung gemacht werden) $\mathrm{r}=0,6084$ und $p=0,0274$ und zwischen Schmerzintensität und Beeinträchtigung der Lebensqualität mit einem $r=0,5452$ und $p=0,0438$.

Es zeigte sich keine signifikante Korrelation zwischen der Nidusgröße und den Schmerzintensitäten.

Eine Patientin (• Tab. 1, Patient Nr. 7), bei der eine RFA eines OO im BWK 12 erfolgreich durchgeführt wurde, stellte sich nach 11 Monaten erneut in unserer Klinik mit ausgeprägten Schmerzen in der Wirbelsäule (Schmerzintensitäten in der Befragung bei 10) vor. Das Wirbelsäulen-MRT sowie die Knochenszintigraphie konnten ein OO-Rezidiv im behandelten BWK 12 und einen Band- scheibenvorfall ausschließen. Die neurologische Untersuchung ergab den Befund einer Wurzelaffektion im Bereich L3-S1 links, DD pseudoradikuläres Syndrom Demzufolge wurden die in der Befragung geäußerten Schmerzen nicht auf ein Rezidiv des OO zurückgeführt. Die Patienten 5 und 9 ( $\mathbf{0}$ Tab. 1) gaben in der Nachuntersuchung geringe Schmerzen ( 2 in der VAS) in der RFA-Region an, mit geringer bzw. fehlender Beeinträchtigung der Lebensqualität oder Leistung In den Kontrollbildgebungen zeigten sich geringe degenerative Veränderungen im linken Hüftgelenk und Kniegelenk bei Zustand nach OO-RFA im Azetabulum und Tibia.

Zwölf der 14 befragten Patienten gaben an, dass sie sich wieder einer RFA bei bestehendem $\mathrm{OO}$ unterziehen würden. Die verbleibenden 2 Patienten machten darüber keine Angaben.

\section{Diskussion}

Die vorliegende Arbeitzeigt, dass die RFA von $\mathrm{OO}$ auch in unterschiedlichen Lokalisationen ein sicheres Verfahren mit sehr guten technischen und klinischen Erfolgsraten ist. Da die Indikation für die Therapie primär in Abhängigkeit von der bestehenden Schmerzsymptomatik gestellt wird, wurden insbesondere die Reduktion der Schmerzen und die Verbesserung der Lebensqualität nach der Behandlung bewertet.

Die CT-gestützte RFA zur minimalinvasiven Behandlung des symptomatischen OO ist die Therapieoption der ersten Wahl [15]. Unsere Ergebnisse mit $100 \%$ technischem Erfolg und 96,3\% primärem bzw. $100 \%$ sekundärem kli- 


\begin{tabular}{|c|c|c|c|c|c|c|}
\hline Pat. Nr. & Geschl. & Alter & $\begin{array}{l}\text { Nidus- } \\
\text { durch- } \\
\text { messer } \\
(\mathrm{mm})\end{array}$ & $\begin{array}{l}\text { Lokali- } \\
\text { sation }\end{array}$ & Seite & $\begin{array}{l}\text { Rezidiv- } \\
\text { Follow- } \\
\text { up } \\
\text { (Tage) }\end{array}$ \\
\hline 1 & W & 23 & 8 & Femur & $\mathrm{Li}$ & 2040 \\
\hline 2 & $M$ & 53 & 3 & Femur & $\mathrm{Li}$ & 1850 \\
\hline 3 & M & 20 & 15 & $\begin{array}{l}\text { Wirbelbogen } \\
\text { BWK } 11\end{array}$ & $\operatorname{Re}$ & 1170 \\
\hline 4 & W & 64 & 4 & Femur & $\mathrm{Li}$ & 900 \\
\hline 5 & $M$ & 32 & 13 & Azetabulum & $\mathrm{Li}$ & 750 \\
\hline 6 & M & 19 & 6 & $\begin{array}{l}\text { Wirbelbogen } \\
\text { BWK } 4\end{array}$ & $\mathrm{Li}$ & 360 \\
\hline 7 & W & 23 & 9 & Korpus BWK 12 & $\mathrm{Li}$ & 360 \\
\hline 8 & W & 22 & 6 & Os naviculare & $\operatorname{Re}$ & 300 \\
\hline 9 & W & 20 & 5 & Tibia & $\mathrm{Li}$ & 300 \\
\hline 10 & $M$ & 15 & 4 & Femur & $\operatorname{Re}$ & 60 \\
\hline 11 & $M$ & 34 & 4 & Femur & $\operatorname{Re}$ & 60 \\
\hline 12 & M & 21 & 4 & Femur & $\mathrm{Li}$ & 60 \\
\hline 13 & M & 11 & 6 & Femur & $\operatorname{Re}$ & 60 \\
\hline 14 & W & 16 & 7 & Femur & $\operatorname{Re}$ & 60 \\
\hline 15 & M & 13 & 8 & Femur & $\operatorname{Re}$ & 1470 \\
\hline 16 & M & 16 & 6 & $\begin{array}{l}\text { Wirbelbogen } \\
\text { LWK } 5\end{array}$ & $\mathrm{Li}$ & 1350 \\
\hline 17 & M & 21 & 5 & Femur & $\mathrm{Li}$ & 7 \\
\hline 18 & M & 21 & 4 & Femur & $\mathrm{Li}$ & 450 \\
\hline 19 & $M$ & 21 & 7 & Caput humeri & $\mathrm{Li}$ & 930 \\
\hline 20 & M & 28 & 6 & Ulna & $\mathrm{Li}$ & 7 \\
\hline 21 & W & 13 & 12 & Tibia & $\operatorname{Re}$ & 390 \\
\hline 22 & M & 34 & 3 & Caput humeri & $\operatorname{Re}$ & 240 \\
\hline 23 & M & 23 & 2 & Digitus IV & $\operatorname{Re}$ & 18 \\
\hline 24 & M & 27 & 3 & Pedikel BWK 9 & $\operatorname{Re}$ & 2 \\
\hline \multirow[t]{2}{*}{25} & W & 4 & 3 & Tibia & $\operatorname{Re}$ & - \\
\hline & W & 5 & 3 & Tibia & $\operatorname{Re}$ & Rezidiv 105 \\
\hline 26 & M & 18 & 7 & Tibia & $\mathrm{Re}$ & 2 \\
\hline
\end{tabular}

nischem Erfolg decken sich mit den Angaben in der Literatur $[4,11]$.

Auch die Ergebnisse der pathohistologischen Untersuchungen sind gut mit den Angaben in der Literatur vereinbar, die von einer Aussagekraft der Befunde von etwa $50 \%$ berichten [14]. Die von uns eingesandten Gewebeproben zeigten nur bei $n=2$ ein OO und bei $n=15$ war der Befund vereinbar mit einem OO (gesamt $77 \%$ ). Bei eindeutiger Klinik und passendem Bildbefund ist die Sinnhaftigkeit der histologischen Untersuchung damit zu hinterfragen. Jedoch sollte bei einer OOuntypischen Klinik oder uneindeutigem
Bildbefund nicht auf die Probeentnahme verzichtet werden.

In unserer Untersuchung wurden zwei klinische Komplikationen bei insgesamt 27 Ablationen registriert (7\%). Dies liegt im Bereich der in der Literatur zu findenden Komplikationsraten, z. B. von Bruners et al. [2]. Die in unserer Studie aufgetretene Weichteilinfektion blieb ohne Langzeitfolgen für den Patienten. Bei der Fraktur des Os naviculare nach erfolgter RFA wurde der Verdacht auf eine Osteonekrose geäußert. Es bleibtjedoch unklar, ob es sich hierbei um eine Komplikation der Behandlung handelte oder ob die- se eine Folge des OO bzw. OO-Rezidiv ist und auch ohne Behandlung aufgetreten wäre. Die aggressive Ablation von $\mathrm{OO}$ in kleinen Knochen sollte allerdings unserer Meinung nach somit einer strengen Indikationsprüfung unterliegen und mögliche Alternativen wie z. B. eine alleinige Ausbohrung oder die chirurgische Kürettage in Erwägung gezogen werden. Die Patienten mit einem solchen Befund müssen über diese Art der Komplikation zusätzlich aufgeklärt werden.

Der Bruch eines manuellen Bohrers hatte zwar zu keinem klinischen Nachteil für den Patienten, aber zu erheblichen intraprozeduralen Schwierigkeiten geführt. Insbesondere OO in langen Röhrenknochen können extrem hart sein und führen bei manuellen Bohrsystemen zu erheblichen Belastungen und $\mathrm{zu}$ einer Verlängerung der Prozedur. Unter anderem infolge dieser Schwierigkeit wurde ein elektrisches Bohrsystem eingeführt, welches in keinem Fall mehr zu einem Materialbruch geführt und die Prozeduren insgesamt auch deutlich beschleunigt hat. Dessen Einsatz kann dringend empfohlen werden.

Obwohl die klinische Symptomatik insbesondere mit Schmerzen die führende Motivation fürdie Behandlung des $\mathrm{OO}$ darstellt, beschäftigen sich nur wenige Arbeiten intensiver mit einer Quantifizierung der Einschränkung der Lebensqualität durch Schmerzen vor und im Verlauf nach bildgesteuerter lokalablativer Therapie. In dieser Arbeit erfolgte die Befragung der Patienten bezüglich ihrer Schmerzen und der damit einhergehenden Beeinträchtigung der Lebensqualität, körperlichen Aktivität und Leistungsfähigkeit zu verschiedenen Zeitpunkten seit dem Auftreten der ersten OO-Symptome. Es konnte gezeigt werden, dass sich die Schmerzintensitäten vor RFA bzw. im Verlauf der konservativen Therapie (Sz1 und Sz2) nicht signifikant änderten. Auch die damit einhergehende $\mathrm{Be}$ einträchtigung der Lebensqualität änderte sich nicht. Die Schmerzintensität korrelierte deutlich mit der Einschränkung der Leistungsfähigkeit, körperlichen Aktivität und letztlich der Lebensqualität. Wie auch von Omlor et al. [10] berichtet, zeigte sich erst nach der RFA eine signifikante Schmerzreduktion. Es konn- 
te gezeigt werden, dass sich im Nachbeobachtungszeitraum die Lebensqualität ohne signifikante Beeinträchtigung durch OO-Symptome normalisiert. Es kann also geschlussfolgert werden, dass die konservative Therapie im längerfristigen Verlauf keine Option für den Patienten bedeutet. Eine schnelle Diagnosesicherung und kausale Behandlung des OO sollte immer angestrebt werden, um die Zeit mit beeinträchtigter Lebensqualität zu verkürzen und letztlich auch unnötige Behandlungen oder Folgeerkrankungen und -kosten zu vermeiden. Des Weiteren besteht die Gefahr, dass die zum Teil sehr langen Verläufe (durchschnittlich 22 Monate nach dem ersten Arztbesuch bis zur RFA) zu einer schmerzbedingten Fehlbelastung des muskuloskeletalen Systems und infolgedessen zu sekundären Schmerzsymptomen führen. Ein Beispiel dafür ist die Patientin mit einem zwar erfolgreich behandelten OO im BWK 12, aber einer im Verlauf aufgetretenen lumbalen Nervenwurzelaffektion bzw. einem pseudoradikulären Syndrom nach jahrelanger Fehlbelastung. Ein weiteres Beispiel ist der Patient mit OO im Azetabulum und der Tibia, der nach erfolgreicher lokaler Behandlung aufgrund haltungsbedingter, degenerativer Veränderungen Gelenkschmerzen entwickelt hat. Abschließend konnte diese Arbeit zeigen, dass auch bei schon lang bestehender OO-typischer Schmerzsymptomatik und bei $\mathrm{OO}$ in atypischen Lokalisationen eine Normalisierung der Lebensqualität durch die minimalinvasive bildgeführte Therapie mit RFA zuverlässig und komplikationsarm möglich ist.

\section{Limitationen}

Limitiert wird die vorliegende Auswertung durch die vergleichsweise geringe Patientenzahl mit $\mathrm{OO}$ in unterschiedlichen Lokalisationen und die retrospektive Datenerfassung. Außerdem wurden die Patienten teilweise zu mehrere Jahre zurückliegenden Schmerzintensitäten und deren Einfluss auf die Lebensqualität und die Aktivität vor und nach der Behandlung befragt.

\section{Fazit für die Praxis \\ - Das 00 präsentiert sich mit typi- scher klinischer Symptomatik und Bildgebung. Die histopathologische Untersuchung dagegen ist nicht zielführend. \\ - Die Behandlung mittels RFA ist ein effizientes und komplikationsarmes Therapieverfahren des 00 in sehr unterschiedlichen Lokalisationen. \\ - Frühzeitige Diagnosestellung und Therapie vermeiden eine schmerzbe- dingte Minderung der Lebensqualität und Fehlbelastung und sekundäre degenerative Veränderungen in angrenzenden Gelenken.}

\section{Korrespondenzadresse}

\section{Seider}

Medizinische Fakultät der Universität Leipzig, Klinik und Poliklinik für Diagnostische und Interventionelle Radiologie, Universitätsklinikum Leipzig AöR

Liebigstraße 20, 04103 Leipzig, Deutschland Daniel.Seider@medizin.uni-leipzig.de

\section{Einhaltung ethischer Richtlinien}

Interessenkonflikt. D. Seider, M. Reinhardt, D. Zajonz, P. Voigt, T. Kahn und M. Moche geben an, dass kein Interessenkonflikt besteht.

Dieser Beitrag beinhaltet keine von den Autoren durchgeführten Studien an Menschen oder Tieren.

\section{Literatur}

1. Assoun J, Richardi G, Railhac JJ et al (1994) Osteoid osteoma. MR imaging versus CT. Radiology 191(1):217-223. https://doi.org/10.1148/ radiology.191.1.8134575

2. Bruners P, Penzkofer T, Günther RW et al (2009) Perkutane Radiofrequenzablation von Osteoidosteomen. Technik und Ergebnisse (Percutaneous radiofrequency ablation of osteoid osteomas: technique and results). Rofo 181(8):740-747. https://doi.org/10.1055/s-0028-1109424

3. Donkol RH, Al-Nammi A, Moghazi K (2008) Efficacy of percutaneous radiofrequency ablation of osteoid osteoma in children. Pediatr Radiol 38(2):180-185. https://doi.org/10.1007/s00247007-0690-z

4. Gebauer B, Collettini F, Bruger C et al (2013) Radiofrequency ablation of osteoid osteomas. Analgesia and patient satisfaction in long-term follow-up. Rofo 184(10):959-966. https://doi.org/ 10.1055/s-0033-1350347

5. Hoffmann R-T, Jakobs TF, Kubisch CH et al (2010) Radiofrequency ablation in the treatment of osteoid osteoma-5-year experience. Eur J Radio 73(2):374-379. https://doi.org/10.1016/j.ejrad. 2008.11.018

6. Husen $M$, Hövel $M$, Jäger $M(2016)$ Das intraartikuläre Osteoidosteom. Eine diagnostische wie therapeutische Herausforderung (The intraarticular osteoid osteoma: A diagnostic and therapeutic challenge). Orthopade 45(6):544-548. https://doi. org/10.1007/s00132-015-3220-y

7. Jaffe HL (1935) Osteoid-Osteoma. Arch Surg 31(5):709. https://doi.org/10.1001/archsurg. 1935 01180170034003

8. Lanza E, Thouvenin Y, Viala P et al (2014) Osteoid osteoma treated by percutaneous thermal ablation. When do we fail? A systematic review and guidelines for future reporting. Cardiovasc Intervent Radiol 37(6):1530-1539. https://doi.org/ 10.1007/s00270-013-0815-8

9. Neumann D, Berka H, Dorn U et al (2012) Followup of thirty-three computed-tomography-guided percutaneous radiofrequency thermoablations of osteoid osteoma. Int Orthop 36(4):811-815. https://doi.org/10.1007/s00264-011-1402-8

10. Omlor G, Merle C, Lehner B et al (2012) CTguided percutaneous radiofrequency ablation in osteoid osteoma. Re-assessments of results with optimized technique and possible pain patterns in mid-term follow-up. Rofo 184(4):333-339. https:// doi.org/10.1055/s-0031-1299121

11. Rehnitz C, Sprengel SD, Lehner B et al (2012) $\mathrm{CT}$-guided radiofrequency ablation of osteoid osteoma and osteoblastoma. Clinical success and long-term follow up in 77 patients. Eur J Radiol 81(11):3426-3434. https://doi.org/10. 1016/j.ejrad.2012.04.037

12. Rehnitz C, Sprengel SD, Lehner B et al (2013) CTguided radiofrequency ablation of osteoid osteoma. Correlation of clinical outcome and imaging features. Diagn Interv Radiol 19(4):330-339. https://doi.org/10.5152/dir.2013.096

13. Rosenthal DI, Hornicek FJ, Wolfe MW et al (1998) Percutaneous radiofrequency coagulation of osteoid osteoma compared with operative treatment. J Bone Joint Surg Am 80(6):815-821

14. Schmidt D, Clasen S, Schaefer JF et al (2011) CT-gesteuerte Radiofrequenz (RF)-Ablation von Osteoidosteomen. Klinische Langzeitergebnisse (CT-guided radiofrequency (RF) ablation of osteoid osteoma: clinical long-term results). Rofo 183(4):381-387. https://doi.org/10.1055/s-00291245998

15. Schnapauff D, Streitparth F, Jöhrens K et al (2015) $\mathrm{CT}$-guided radiofrequency ablation of osteoid osteoma using a novel battery-powered drill. Skeletal Radiol 44(5):695-701. https://doi.org/10 1007/s00256-014-2029-9

16. Sprengel SD, Weber M-A, Lehner B et al (2015) Osteoidosteom. Von der Diagnose zur Behandlung (Osteoidosteoma. From diagnosis to treatment). Radiologe 55(6):479-486. https://doi.org/10. 1007/s00117-014-2805-5

17. Sung K-S, Seo J-G, Shim JS et al (2009) Computed tomography-guided percutaneous radiofrequency thermoablation for the treatment of osteoid osteoma-2 to 5 years follow-up. Int Orthop 33(1):215-218. https://doi.org/10.1007/s00264007-0481-z

18. Weber M-A, Sprengel SD, Omlor GW et al (2015) Clinical long-term outcome, technical success, and cost analysis of radiofrequency ablation for the treatment of osteoblastomas and spinal osteoid osteomas in comparison to open surgical resection. Skeletal Radiol 44(7):981-993. https:// doi.org/10.1007/s00256-015-2139-z 


\section{Zusammenfassung der Arbeit}

Dissertation zur Erlangung des akademischen Grades

doctor medicinae (Dr. med.)

„Analyse der Lebensqualität von Patienten mit Osteoidosteom vor und nach Behandlung mittels Radiofrequenzablation"

eingereicht von

geboren am:

angefertigt an:

betreut von

Torgau, 31. Mai 2019
Daniel Seider

07.03.1980 in Torgau

Medizinische Fakultät der Universität Leipzig

Klinik und Poliklinik für Diagnostische und Interventionelle

Radiologie

Prof. em. Dr. med. habil. Thomas Kahn (ehm. Direktor der Klinik und Poliklinik f. Diagnostische und Interventionelle Radiologie der Universität Leipzig) PD Dr. med. habil. Michael Moche (HELIOS Park-Klinikum Leipzig, Leiter der Klinik f. Interventionelle Radiologie)

Die perkutane Radiofrequenzablation (RFA) ist bereits seit vielen Jahren eine etablierte interventionell-radiologische Methode zur Behandlung von Patienten mit Osteoidosteomen. Sie ist eine sichere und effiziente Alternative zur En-blocResektion und Kürettage. Im Gegensatz zu den offen-chirurgischen Verfahren ist die RFA eine minimalinvasive Methode, bei der die Computertomographie zur genauen Detektion des $\mathrm{OO}$ während der Intervention benutzt wird. Des Weiteren dienen die CT-Bilddaten zur Planung eines geeigneten Zugangsweges sowie der korrekten Platzierung der RFA-Nadel. Die hohen Erfolgs- und niedrigen Komplikationsraten der RFA sind bereits in vielen Publikationen belegt worden. Allerdings gibt es nur wenige Publikationen, in denen die Veränderung der Lebensqualität von Patienten mit $\mathrm{O}$ vor und nach erfolgter RFA untersucht worden sind. 
Ziel dieser Arbeit ist es, die Effektivität der Behandlung von Osteoidosteomen (OO) mittels Radiofrequenzablation (RFA) zu untersuchen. Dabei werden insbesondere die Rezidivrate und die Verbesserung der Lebensqualität durch die Reduktion der Schmerzintensität bewertet.

In einer retrospektiven Analyse wurden 26 Patienten mit stattgehabter RFA eines OO untersucht. 14 Patienten beteiligten sich an einer prospektiven Analyse und beantworteten Fragen eines selbstentwickelten Lebensqualität-Fragebogens. Neben der Aufarbeitung des Fragebogens erfolgte die ergänzende Datenerhebung aus der elektronischen Patientenakte des Universitätsklinikums Leipzig (Krankenhaus- und Radiologieinformationssystem).

Zwischen Juli 2004 bis Oktober 2015 wurden 26 Patienten (m:w 18:8) mit 27 Knochenläsionen mittels CT-gestützter RFA behandelt. Eine Patientin wurde aufgrund eines Rezidivs wiederholt mittels RFA behandelt. Das Durchschnittsalter der Patienten betrug 22,8 \pm 12,7 Jahre (4-64 Jahre). Mehrheitlich wurden die Patienten über hauseigene orthopädische oder kinderchirurgische Ambulanzen in der Radiologie vorgestellt. Meist lagen schon extern angefertigte Röntgenaufnahmen, Computertomographien oder MRT-Bilder vor. Bei uneindeutigen Befunden wurden Bildgebungen ergänzt. Alle Patienten litten an der für das $\mathrm{O}$ typischen klinischen Symptomatik.

Jeder Patient mit klinisch und bildmorphologisch gesichertem $\mathrm{OO}$ wurde im interdisziplinären Board für Knochentumoren vorgestellt. Die Entscheidung zur Behandlung mittels RFA wurde im Konsens getroffen. Im Regelfall erfolgte die stationäre Aufnahme der Patienten am Tag vor der RFA, u.a. um anhand der Blutwerte (Thrombozyten, Quick-Wert) einen suffizienten Gerinnungsstatus sicher zu stellen. 24 Stunden vor der RFA erfolgte die ausführliche Aufklärung. Die Ablation erfolgte in Allgemeinanästhesie und additiver lokaler Anästhesie des Periosts unter streng sterilen Kautelen. Unter bildgestützter Kontrolle mit CT wird der ossäre Zugangsweg etabliert und die RFA-Nadel platziert. Die Radiofrequenzablation folgt dabei meist vom Hersteller vorgegebenen Protokollen. Nach postanästhesiologischer Überwachung kann der Patient auf die Normalstation verlegt und bei Beschwerdefreiheit am ersten postoperativen Tag entlassen werden. Wundkontrollen erfolgen im Rahmen der ambulanten Nachsorge.

Unsere Ergebnisse mit $100 \%$ technischem Erfolg und 96,3\% primärem bzw. $100 \%$ sekundärem klinischem Erfolg decken sich mit den Angaben in der Literatur. Auch 
die Ergebnisse der pathohistologischen Untersuchungen sind mit den Angaben in der Literatur vergleichbar, die von einer Aussagekraft der Befunde von nur etwa $50 \%$ berichten. Bei einer OO-untypischen Klinik oder uneindeutigem Bildbefund sollte unserer Meinung nach nicht auf die Probeentnahme verzichtet werden. Die beiden bei uns registrierten klinischen Komplikationen liegen ebenfalls im Bereich der in der Literatur zu findenden Komplikationsraten.

Die Auswertung der Patientenfragebögen, bezüglich der Schmerzen und der damit einhergehenden Beeinträchtigung der Lebensqualität, körperlichen Aktivität und Leistungsfähigkeit ergab folgende Ergebnisse. Alle Patienten hatten einen hohen Leidensdruck durch Schmerzen. Die Schmerzintensitäten vor RFA bzw. im Verlauf der konservativen Therapie änderten sich nicht signifikant, somit auch nicht die damit einhergehende Beeinträchtigung der Lebensqualität. Dabei korrelierte die Schmerzintensität deutlich mit der Einschränkung der Leistungsfähigkeit und der körperlichen Aktivität und führte dadurch letztlich zur Reduktion der allgemeinen Lebensqualität. Erst nach der RFA zeigte sich eine signifikante Schmerzreduktion. Im Nachbeobachtungszeitraum normalisierte sich die Lebensqualität ohne signifikante Beeinträchtigung durch das OO. Schlussfolgernd kann zusammengefasst werden, dass bei entsprechender klinischer Schmerzsymptomatik eine schnelle Diagnosesicherung durch bildgebende Verfahren erfolgen sollte. Danach sollte sich dann die zeitnahe, kausale Behandlung des $\mathrm{OO}$ primär durch perkutane Ablation anschließen. Dies verkürzt die Zeit mit beeinträchtigter Lebensqualität und reduziert unnötige Behandlungen, Folgeerkrankungen und Kosten. 


\section{Referenzen}

1. Jaffe HL. "Osteoid-Osteoma". Arch Surg 1935; 31(5):709. doi: 10.1001/archsurg.1935.01180170034003.

2. Mankin HJ. Our Debt to Orthopaedic Pathologists [Stand: 13.03.2019]. Verfügbar unter: http://www.orthojournalhms.org/volume9/manuscripts/ms21.pdf.

3. Adler C-P. Knochenkrankheiten: Diagnostik makroskopischer, histologischer und radiologischer Strukturveränderungen des Skeletts. 2. Auflage. Berlin, Heidelberg, s.l.: Springer Berlin Heidelberg; 1998. Verfügbar unter: http://dx.doi.org/10.1007/978-3-662-12160-3.

4. Jundt G. Neues zur WHO-Klassifikation der Knochentumoren. Pathologe 2018; 39(2):107-16. doi: 10.1007/s00292-017-0396-4.

5. Schajowicz F. Tumors and Tumorlike Lesions of Bone: Pathology, Radiology, and Treatment. 2nd edition. Berlin, Heidelberg: Springer Berlin Heidelberg; 1994.

6. Schajowicz F. Histological typing of bone tumours. 2. ed. Berlin: Springer; 1993. (International histological classification of tumours; Bd. 6).

7. Hogendoorn PCW. Bone: Osteoid Osteoma. Atlas of Genetics and Cytogenetics in Oncology and Haematology 2011; (1). doi: 10.4267/2042/44671.

8. Jobke B, Werner M. Osteogene Tumoren. Radiologe 2016; 56(6):489-506. doi: 10.1007/s00117-016-0119-5.

9. Schulman L, Dorfman HD. Nerve fibers in osteoid osteoma. J Bone Joint Surg Am 1970; 52(7):1351-6.

10. Freyschmidt J, Ostertag HM, Jundt G. Knochentumoren mit Kiefertumoren: Klinik - Radiologie - Pathologie. 3. Auflage. Berlin, Heidelberg: Springer-Verlag Berlin Heidelberg; 2010. Verfügbar unter: http://site.ebrary.com/lib/alltitles/docDetail.action?doclD=10447031.

11. Kayser M, Muhr G. Eighteen-year anamnesis of osteoid osteoma - a diagnostic problem? Arch Orthop Trauma Surg 1988; 107(1):27-30.

12. Assenmacher S, Voggenreiter G, Klaes W, Nast-Kolb D. Das Osteoidosteom ein diagnostisches und therapeutisches Problem? Chirurg 2000; 71(3):319-25. 
13. Campanacci M, Ruggieri P, Gasbarrini A, Ferraro A, Campanacci L. Osteoid osteoma. Direct visual identification and intralesional excision of the nidus with minimal removal of bone. J Bone Joint Surg Br 1999; 81(5):814-20.

14. Heuck A, Stäbler A, Wörtler K, Steinborn M. Gutartige knochenbildende Tumoren. Radiologe 2001; 41(7):540-7. doi: 10.1007/s001170170144.

15. Rand JA, Sim FH, Unni KK. Two osteoid-osteomas in one patient. A case report. J Bone Joint Surg Am 1982; 64(8):1243.

16. Alcalay M, Clarac JP, Bontoux D. Double osteoid-osteoma in adjacent carpal bones. A case report. J Bone Joint Surg Am 1982; 64(5):779-80.

17. Knöfler EW. Osteoid-Osteom oder Corticalis-Osteoid? Arch orthop Unfall-Chir 1963; 55(4):462-75. doi: 10.1007/BF01880757.

18. Leonhardt J, Bastian L, Rosenthal H, Laenger F, Wippermann B.

Posttraumatisches Osteoidosteom. Der Unfallchirurg 2001; 104(6):553-6. doi: 10.1007/s001130170120.

19. Lindner NJ, Scarborough M, Ciccarelli JM, Enneking WF. Die CT-gesteuerte Thermokoagulation des Osteoidosteoms im Vergleich zu traditionellen Verfahren. Z Orthop Unfall 1997; 135(6):522-7. doi: 10.1055/s-2008-1039739.

20. Golding JS. The natural history of osteoid osteoma; with a report of twenty cases. J Bone Joint Surg Br 1954; 36-B(2):218-29.

21. Esquerdo J, Fernandez CF, Gomar F. Pain in osteoid osteoma: Histological facts. Acta Orthop Scand 1976; 47(5):520-4.

22. Kattapuram SV, Kushner DC, Phillips WC, Rosenthal DI. Osteoid osteoma: An unusual cause of articular pain. Radiology 1983; 147(2):383-7. doi: 10.1148/radiology.147.2.6836118.

23. Norman A, Abdelwahab IF, Buyon J, Matzkin E. Osteoid osteoma of the hip stimulating an early onset of osteoarthritis. Radiology 1986; 158(2):417-20. doi: 10.1148/radiology.158.2.3941866.

24. Dahlin DC, Johnson EW. Giant osteoid osteoma. J Bone Joint Surg Am 1954; 36 $A(3): 559-72$. 
25. Lucas DR, Unni KK, McLeod RA, O'Connor MI, Sim FH. Osteoblastoma:

Clinicopathologic study of 306 cases. Hum Pathol 1994; 25(2):117-34.

26. Greenspan A, Beltran J. Skelettradiologie: Orthopädie, Traumatologie, Rheumatologie, Onkologie. 6. Auflage. München, Deutschland: Elsevier; 2017. (German medical collection; Bd. 39). Verfügbar unter: http://www.sciencedirect.com/science/book/9783437230646.

27. Winkelmann W. Tumoren, tumorähnliche Erkrankungen. 1. Auflage. s.l.: Thieme; 2005. Verfügbar unter: http://dx.doi.org/10.1055/b-001-2154.

28. Allen SD, Saifuddin A. Imaging of intra-articular osteoid osteoma. Clin Radiol 2003; 58(11):845-52.

29. Berning W, Freyschmidt J, Wiens J. Zur perkutanen Therapie des Osteoidosteoms. Der Unfallchirurg 1997; 100(7):536-40.

30. Orth P, Kohn D. Diagnostik und Therapie des Osteoidosteoms. Orthopäde 2017; 46(6):510-21. doi: 10.1007/s00132-017-3428-0.

31. Helms CA, Hattner RS, Vogler JB. Osteoid osteoma: Radionuclide diagnosis. Radiology 1984; 151(3):779-84. doi: 10.1148/radiology.151.3.6232642.

32. Steinberg GG, Coumas JM, Breen T. Preoperative localization of osteoid osteoma: A new technique that uses CT. Am J Roentgenol 1990; 155(4):883-5. doi: 10.2214/ajr.155.4.2119126.

33. Gamba JL, Martinez S, Apple J, Harrelson JM, Nunley JA. Computed tomography of axial skeletal osteoid osteomas. Am J Roentgenol 1984; 142(4):769-72. doi: 10.2214/ajr.142.4.769.

34. Liu PT, Chivers FS, Roberts CC, Schultz CJ, Beauchamp CP. Imaging of osteoid osteoma with dynamic gadolinium-enhanced MR imaging. Radiology 2003; 227(3):691-700. doi: 10.1148/radiol.2273020111.

35. Davies M, Cassar-Pullicino VN, Davies AM, McCall IW, Tyrrell PNM. The diagnostic accuracy of MR imaging in osteoid osteoma. Skeletal Radiol 2002; 31(10):559-69. doi: 10.1007/s00256-002-0546-4.

36. Ilyas I, Younge DA. Medical management of osteoid osteoma. Can J Surg 2002; 45(6):435-7. 
37. Karow T LR. Allgemeine und Spezielle Pharmakologie und Toxikologie; s.I. 2010.

38. Yildiz Y, Bayrakci K, Altay M, Saglik Y. Osteoid osteoma: The results of surgical treatment. Int Orthop 2001; 25(2):119-22.

39. Powell MF, DiNobile D, Reddy AS. C-arm fluoroscopic cone beam CT for guidance of minimally invasive spine interventions. Pain Physician 2010; 13(1):51-9.

40. Hempfing A, Hoffend J, Bitsch RG, Bernd L. The indication for gamma probeguided surgery of spinal osteoid osteomas. Eur Spine J 2007; 16(10):1668-72. doi: 10.1007/s00586-007-0388-9.

41. Pratali R, Zuiani G, Inada M, Hanasilo C, Reganin L, Etchebehere E et al. Open resection of osteoid osteoma guided by a gamma-probe. Int Orthop 2009; 33(1):219-23. doi: 10.1007/s00264-008-0552-9.

42. Doyle T, King K. Percutaneous removal of osteoid osteomas using CT control. Clin Radiol 1989; 40(5):514-7. doi: 10.1016/S0009-9260(89)80268-5.

43. Mazoyer JF, Kohler R, Bossard D. Osteoid osteoma: CT-guided percutaneous treatment. Radiology 1991; 181(1):269-71. doi:

10.1148/radiology.181.1.1887046.

44. Erdtmann B, Duda SH, Pereira P, Heim BK, Claussen CD. CT-gesteuerte Therapie des Osteoidosteoms durch Nidusbohrung: Klinische Langzeitergebnisse. Rofo 2001; 173(8):708-13. doi: 10.1055/s-2001-16402.

45. Adam G, Keulers P, Vorwerk D, Heller KD, Füzesi L, Günther RW. Perkutane CTgesteuerte Behandlung von Osteoid-osteomen: Kombiniertes Vorgehen mit einem Hohlbohrer und nachfolgender Athanolinjektion. Rofo 1995; 162(3):232-5. doi: 10.1055/s-2007-1015871.

46. Gangi A, Dietemann JL, Guth S, Vinclair L, Sibilia J, Mortazavi R et al. Percutaneous laser photocoagulation of spinal osteoid osteomas under CT guidance. Am J Neuroradiol 1998; 19(10):1955-8.

47. Witt JD, Hall-Craggs MA, Ripley P, Cobb JP, Bown SG. Interstitial laser photocoagulation for the treatment of osteoid osteoma. J Bone Joint Surg $\mathrm{Br}$ 2000; 82-B(8):1125-8. doi: 10.1302/0301-620X.82B8.0821125. 
48. Rosenthal DI, Alexander A, Rosenberg AE, Springfield D. Ablation of osteoid osteomas with a percutaneously placed electrode: A new procedure. Radiology 1992; 183(1):29-33. doi: 10.1148/radiology.183.1.1549690.

49. Pinto $\mathrm{CH}$, Taminiau AHM, Vanderschueren GM, Hogendoorn PCW, Bloem JL, Obermann WR. Technical considerations in CT-guided radiofrequency thermal ablation of osteoid osteoma: Tricks of the trade. Am J Roentgenol 2002; 179(6):1633-42. doi: 10.2214/ajr.179.6.1791633.

50. Rosenthal DI, Hornicek FJ, Torriani M, Gebhardt MC, Mankin HJ. Osteoid osteoma: Percutaneous treatment with radiofrequency energy. Radiology 2003; 229(1):171-5. doi: 10.1148/radiol.2291021053.

51. Vanderschueren GM, Taminiau AHM, Obermann WR, van den Berg-Huysmans AA, Bloem JL. Osteoid osteoma: Factors for increased risk of unsuccessful thermal coagulation. Radiology 2004; 233(3):757-62. doi: 10.1148/radiol.2333031603. 


\section{Erklärung zur eigenständigen Abfassung der Arbeit}

Hiermit erkläre ich, dass ich die vorliegende Arbeit selbstständig und ohne unzulässige Hilfe oder Benutzung anderer als der angegebenen Hilfsmittel angefertigt habe. Ich versichere, dass Dritte von mir weder unmittelbar noch mittelbar eine Vergütung oder geldwerte Leistungen für Arbeiten erhalten haben, die im Zusammenhang mit dem Inhalt der vorgelegten Dissertation stehen, und dass die vorgelegte Arbeit weder im Inland noch im Ausland in gleicher oder ähnlicher Form einer anderen Prüfungsbehörde zum Zweck einer Promotion oder eines anderen Prüfungsverfahrens vorgelegt wurde. Alles aus anderen Quellen und von anderen Personen übernommene Material, das in der Arbeit verwendet wurde oder auf das direkt Bezug genommen wird, wurde als solches kenntlich gemacht. Insbesondere wurden alle Personen genannt, die direkt an der Entstehung der vorliegenden Arbeit beteiligt waren. Die aktuellen gesetzlichen Vorgaben in Bezug auf die Zulassung der klinischen Studien, die Bestimmungen des Tierschutzgesetzes, die Bestimmungen des Gentechnikgesetzes und die allgemeinen Datenschutzbestimmungen wurden eingehalten. Ich versichere, dass ich die Regelungen der Satzung der Universität Leipzig zur Sicherung guter wissenschaftlicher Praxis kenne und eingehalten habe.

Torgau, den 31.05.2019

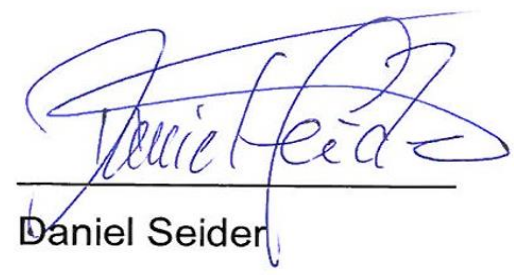




\section{Darstellung des eigenen Beitrages an der Publikation}

Zu der, dieser Dissertation zugrundeliegenden, Publikation "Analyse der Lebensqualität von Patienten mit Osteoidosteom vor und nach Behandlung mittels Radiofrequenzablation. D. Seider, M. Reinhardt, D. Zajonz, P. Voigt, Th. Kahn, M. Moche. Der Chirurg, DOI 10.1007/s00104-019-0818-3, habe ich Daniel Seider folgende Beiträge geleistet:

- Konzeption und Planung der Patentenbefragung und Analyse

- Durchführung der Literaturrecherche

- Datenrecherche aus RIS und SAP

- Grundlegende statistische Auswertungen

- Erstellung und Korrektur des Publikationsmanuskriptes

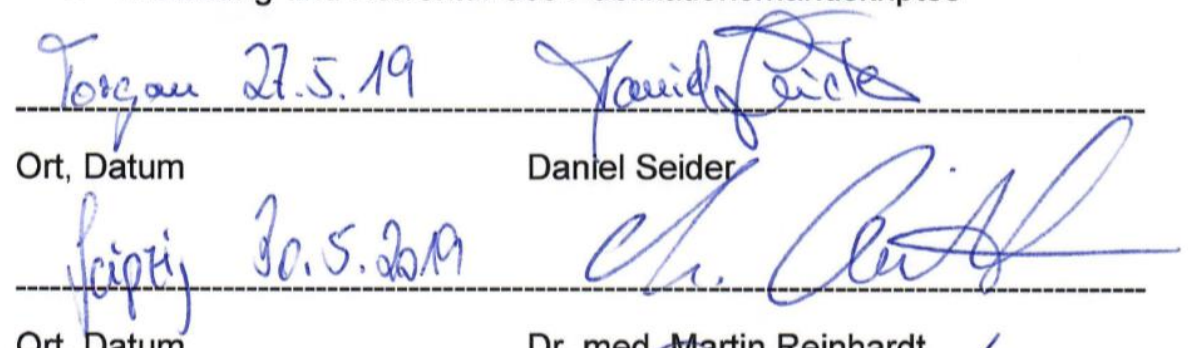

Ort, Datum

Dr. med. Martin Reinhardt

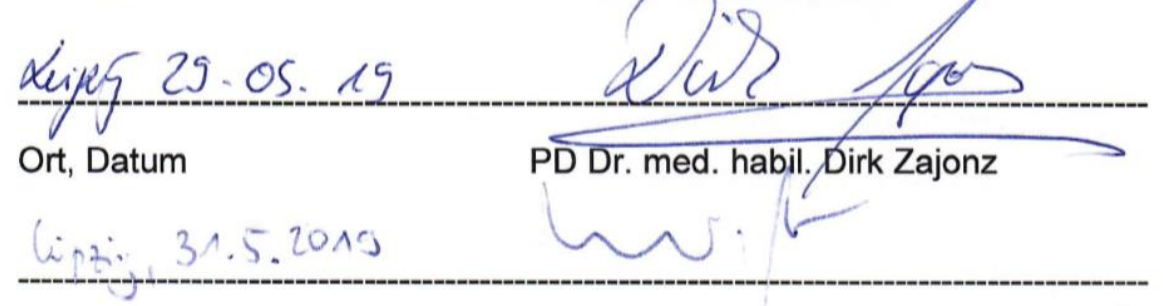

Ort, Datum

Ort, Datum 2 Prof. en. Dr. med. habil. Thomas Kahn

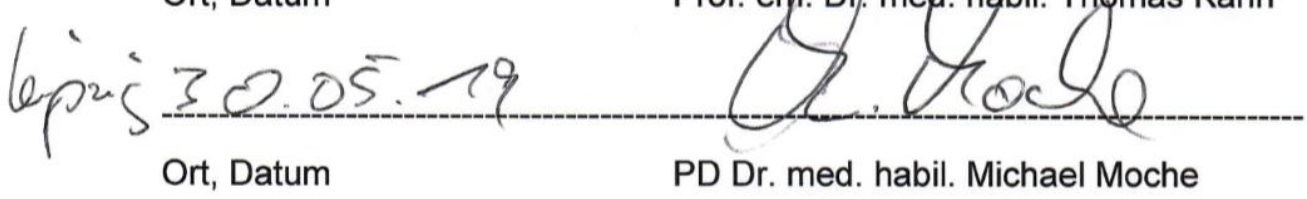


10. Curriculum Vitae 


\section{Bisherige Publikationen}

Subtotal Segment Resection, Augmenting Angle-Stable Plate Osteosynthesis and Allogenic Bone Graft as Treatment Option for Juxtacortical Osteosarcoma in the Knee Region.

Zajonz D, Pfraenger JR, Dubitzki S, Panzert S, Seider D, Lingscheidt T, Hammer N, Prietzel T.

Z Orthop Unfall. 2015 Jun; 153(3):299-305

RFA-cut: Semi-automatic segmentation of radiofrequency ablation zones with and without needles via optimal s-t-cuts.

Egger J, Busse H, Brandmaier P, Seider D, Gawlitza M, Strocka S, Voglreiter P, Dokter M, Hofmann M, Kainz B, Chen X, Hann A, Boechat P, Yu W, Freisleben B, Alhonnoro T, Pollari M, Moche M, Schmalstieg D.

Conf Proc IEEE Eng Med Biol Soc. 2015; 2015:2423-9

Interactive Volumetry of Liver Ablation Zones.

Egger J, Busse H, Brandmaier P, Seider D, Gawlitza M, Strocka S, Voglreiter P, Dokter M, Hofmann M, Kainz B, Hann A, Chen X, Alhonnoro T, Pollari M,

Schmalstieg D, Moche M.

Sci Rep. 2015 Oct 20;5: 15373

Immunohistochemical and radiological characterization of wound healing in porcine liver after radiofrequency ablation.

Stadlbauer V, Lang-Olip I, Leber B, Mayrhauser U, Koestenbauer S, Tawdrous M, Moche M, Sereinigg M, Seider D, Iberer F, Wiederstein-Grasser I, Portugaller RH, Stiegler $P$.

Histol Histopathol. 2016 Jan; 31(1):115-29.

Navigated MRI-guided liver biopsies in a closed-bore scanner: experience in 52 patients.

Moche M, Heinig S, Garnov N, Fuchs J, Petersen TO, Seider D, Brandmaier P, Kahn T, Busse $\mathrm{H}$.

Eur Radiol. 2016 Aug; 26(8):2462-70

A prospective development study of software-guided radio-frequency ablation of primary and secondary liver tumors: Clinical intervention modelling, planning and proof for ablation cancer treatment (ClinicIMPPACT).

Reinhardt M, Brandmaier P, Seider D, Kolesnik M, Jenniskens S, Sequeiros RB, Eibisberger M, Voglreiter P, Flanagan R, Mariappan P, Busse H, Moche M;

ClinicIMPPACT Study Group.

Contemp Clin Trials Commun. 2017 Aug 18; 8: 25-32 
12. Danksagung 